\title{
Norm coherence for descent of level structures on formal deformations
}

\author{
YIFEI ZHU
}

\begin{abstract}
We give a formulation for descent of level structures on deformations of formal groups, and study the compatibility between the descent and a norm construction. Under this framework, we generalize Ando's construction of $\mathrm{H}_{\infty}$ complex orientations for Morava E-theories associated to Honda formal group laws over $\mathbb{F}_{p}$. We show the existence and uniqueness of such an orientation for any Morava E-theory associated to a formal group law over an algebraic extension of $\mathbb{F}_{p}$ and, in particular, orientations for a family of elliptic cohomology theories. These orientations correspond to coordinates on deformations of formal groups which are compatible with norm maps along descent.
\end{abstract}

\section{Introduction}

1.1 Algebraic motivations and statement of results Let $R$ be a commutative ring with 1 and let $A$ be an algebra over $R$. Suppose that, as an $R$-module, $A$ is finitely generated and free. The norm of $A$ is a map $A \rightarrow R$ which sends $a$ to $\operatorname{det}(a \cdot)$, the determinant of multiplication by $a$ as an $R$-linear transformation on $A$. It is multiplicative but not additive in general. Such norms appear as an important ingredient in various contexts: arithmetic moduli of elliptic curves [Katz-Mazur1985, §1.8, §7.7], actions of finite group schemes on abelian varieties [Mumford2008, §12], isogenies of one-parameter formal Lie groups over $p$-adic integer rings [Lubin1967, §1]. These norm maps are closely related to construction of quotient objects.

It is the purpose here to examine an interaction between norms and the corresponding subobjects - more precisely, a functorial interaction with chains of subobjects-in the context of Lubin and Tate's formal deformations [Lubin-Tate1966]. The functoriality amounts to descent of "level structures" on deformations (see $\S 6$ and $\S 8$ ). In this paper, a level structure on a formal group is a choice of finite subgroup scheme, from which we obtain a quotient morphism of formal groups. A norm map between their rings of 
functions then gets involved in making this quotient morphism into a homomorphism of formal group laws (2.3). This norm construction is compatible with successive quotient along a chain of subgroups.

On the other hand, given a deformation over a $p$-adic integer ring, there is a canonical (i.e., coordinate-free) descent of level structures via Lubin and Tate's universal deformations. Strickland studied the representability of this moduli problem [Strickland1997] so that the descent can be realized as canonical lifts of Frobenius morphisms (5.6).

Our main result shows the existence and uniqueness of deformations of formal group laws on which the canonical lifts of Frobenius coincide with quotient homomorphisms from the norm construction. We call these deformations norm-coherent (see $\S 6$, specifically Definition 6.17).

Theorem 1.2 (cf. Theorem 7.22 and Proposition 7.1) Let $k$ be an algebraic extension of $\mathbb{F}_{p}, R$ a complete local ring with residue field containing $k, G$ a formal group law over $k$ of finite height, and $F$ a deformation of $G$ to $R$. There exists a unique formal group law $F^{\prime}$ over $R, \star$-isomorphic to $F$, which is norm-coherent. Moreover, when $F$ is a Lubin-Tate universal deformation, $F^{\prime}$ is functorial under base change of $G / k$, under $k$-isogeny out of $G$, and under $k$-Galois descent.

Remark 1.3 In the context of local class field theory via Lubin-Tate theory, Coleman's norm operator is used to compute norm groups [Coleman1979, Theorem 11]. Walker observed its similarity to the norm construction above [Walker2008, Chapter 5]. Specifically, he reformulated the norm-coherence condition (for a special case) in terms of a particular way in which Coleman's norm operator acts [ibid., 5.0.10]. It would be interesting to understand this connection in view of Theorem 1.2.

1.4 Topological motivations and statement of results The relevance to topology (and, further, to geometry and mathematical physics) of this functorial interaction between norms and finite formal subgroup schemes lies, for instance, in having highly coherent multiplications for genera. These are cobordism invariants of manifolds; such multiplications refine the invariants by reflecting symmetries of the geometry.

A prominent example is the Witten genus for string manifolds, which takes values in the ring of integral modular forms of level 1. Motivated by this, Hopkins and his collaborators developed highly-structured multiplicative orientations (i.e., genera of families of manifolds) for elliptic cohomology theories and for a universal theory of topological modular forms [Hopkins1995, Hopkins2002]. In particular, in [Ando-Hopkins-Strickland2004], they showed that their sigma orientation 
$M U\langle 6\rangle \rightarrow \boldsymbol{E}$ for any elliptic cohomology $\boldsymbol{E}$ is $\mathrm{H}_{\infty}$, a commutativity condition on its multiplicative structure (2.9). Their analysis of this $\mathrm{H}_{\infty}$-structure was based on [Ando1995, Ando1992] where the algebraic condition of norm coherence had made a first appearance.

Theorem 1.2 produces $\mathrm{H}_{\infty} M U\langle 0\rangle$-orientations for a family of generalized cohomology theories called Morava E-theories (2.7) including those treated by Ando and by Ando, Hopkins, Strickland.

Theorem 1.5 (cf. Corollary 8.17) Let $k$ and $G$ be as in Theorem 1.2. For the form of Morava E-theory associated to $G / k$, there exists a unique $M U\langle 0\rangle$-orientation that is an $H_{\infty}$ map.

Remark 1.6 Rezk reminded us that the sigma orientations do not factor through these $\mathrm{H}_{\infty} M U\langle 0\rangle$-orientations (8.1). On the other hand, the coefficient ring of an E-theory (of height 2) is a certain completion of a ring of modular forms. In [Zhu2015a], as a first step, we related its elements to certain quasimodular forms (and to mock modular forms) via Rezk's logarithmic operations; see also [Rezk2016, remarks following Theorem 1.29]. Given Theorem 1.5, it would be interesting to have more exotic manifold invariants. In particular, we may investigate an analogue of the modular invariance of a sigma orientation [Ando-Hopkins-Strickland2001, 1.3] in view of the uniqueness above.

Remark 1.7 A natural question is whether there exist $\mathrm{E}_{\infty}$ complex orientations for Morava E-theories and, more specifically, whether the orientation in Theorem 1.5 rigidifies to be an $\mathrm{E}_{\infty}$ map. See [Hopkins-Lawson2016] for recent progress on $\mathrm{E}_{\infty}$ complex orientations, where the norm-coherence condition comes up.

Finally, the expositions in [Rezk2015] and [Rezk2016, esp. §4] provide some other perspectives. See also [Strickland, esp. §29].

1.8 Outline of the paper In $\S 2$, we recall some basic concepts from the theory of formal groups and homotopy theory, particularly quotient of formal groups (2.3), and set their notation.

In $\S 3$, following a suggestion of Rezk, we introduce an enlarged category of formal groups (cf. [Katz-Mazur1985, §4.1]). This viewpoint will be helpful in clarifying deformations of Frobenius $(5.2,5.9)$, descent of level structures $(6.7,6.10)$, the normcoherence condition (6.16), and functoriality of norm coherence (7.21). 
$\S 4$ and $\S 5$ give an account for the theorems of Lubin, Tate (4.6) and of Strickland (5.5) on deformations of formal groups. Our formulation follows Rezk's (e.g., in [Rezk2014, $\S 4])$ but emphasizes formal group laws. The purpose of these two sections is to provide a detailed exposition as well as a precise setup that is crucial for the notion of norm coherence to follow in desired generality.

In $\S 6$, we introduce the central notion of this paper, norm coherence (6.15-6.19), building on Ando's framework [Ando1995, §2]. We then generalize his theorem and prove Theorem 1.2 in $§ 7$. Our main results are Proposition 7.1 and Theorem 7.22, the latter stated in a form suggested by Rezk.

$\S 8$ discusses the corresponding topological result for complex orientations, with (8.1) introducing further background on work of Ando, of Ando, Hopkins, Strickland, and of Ando, Strickland. In (8.3-8.15), we compare the setup for our results above with Ando, Hopkins, and Strickland's descent data and norm maps [Ando-Hopkins-Strickland2004, Parts 1 and 3]. The purpose is to continue the exposition from $\S 5$ while proving Theorem 1.5.

1.9 Acknowledgements This paper originated from a referee's comment on the choice of coordinates in one of my earlier works. I thank the anonymous referee for their demand for precision on specifics.

I thank Anna Marie Bohmann, Paul Goerss, Fei Han, Michael Hill, Tyler Lawson, Niko Naumann, and Eric Peterson for helpful discussions. I thank Zhen Huan for the quick help with locating a reference.

I learned most of what I know about norm coherence and related questions from Charles Rezk. A good deal of the theory presented here was developed in discussions with him, including "norm-coherent." The term is my choice over the synonym "Ando" and it is Matthew Ando who originally discovered this condition in algebra and applied it to topology.

I thank Eric Peterson for the feedback on a draft of this paper, and for explaining to me the results and methods of his joint work with Nathaniel Stapleton, which gives a different approach to questions considered here.

1.10 Conventions Unless explicitly noted, we fix a prime $p$ throughout this paper.

We often omit the symbol $\operatorname{Spf}$ and simply write $R$ for $\operatorname{Spf} R$ when it appears as a base scheme. In particular, $\beta^{*}$ means base change from $R$ to $S$ along $\beta: R \rightarrow S$, understood as $\beta$ : $\operatorname{Spf} S \rightarrow \operatorname{Spf} R$. 
We also write $\psi^{*}$ for the pullback of functions along a morphism $\psi$ of schemes.

\section{General notions}

2.1 Formal groups, coordinates, and formal group laws Let $R$ be a complete local ring with residue characteristic $p>0$. A formal group $\mathcal{G}$ over $R$ is a group object in the category of formal $R$-schemes. In this paper, all formal groups will be commutative, one-dimensional, and affine. They can be viewed as covariant functors from the category of complete local $R$-algebras (and local homomorphisms) to the category of abelian groups.

A coordinate $x$ on $\mathcal{G}$ is a natural isomorphism $\mathcal{G} \stackrel{\sim}{\rightarrow} \hat{\mathbb{A}}_{R}^{1}$ of functors to pointed sets. It gives an isomorphism $\Gamma\left(\mathcal{G}, \mathcal{O}_{G}\right) \cong R \llbracket x \rrbracket$ of augmented $R$-algebras, as well as a trivialization of the ideal sheaf $\mathcal{I}_{G}(O)=\mathcal{O}_{G}(-O)$ of functions on $\mathcal{G}$ which vanish at the identity section $O$. Here and throughout the paper, we remove the calligraphic effect of the notation for a formal group whenever it appears as a subscript. We will also simply write $\mathcal{O}_{G}$ for the ring of global sections of $\mathcal{O}_{G}$, and similarly for other sheaves.

A (one-dimensional commutative) formal group law $F$ over $R$ is a formal power series in two variables $t_{1}$ and $t_{2}$ with coefficients in $R$, often written $t_{1}+{ }_{F} t_{2}$, which satisfies a set of abelian-group-like axioms. In particular, the above data of $\mathcal{G}$ and $x$ determines a formal group law $G=G_{x}$ such that

$$
x\left(P_{1}\right) \underset{G}{+} x\left(P_{2}\right)=x\left(P_{1}+P_{2}\right)
$$

for any $R$-points $P_{1}$ and $P_{2}$ on $\mathcal{G}$ (where we identify an $R$-point on $\hat{\mathbb{A}}_{R}^{1}$ with an element in the maximal ideal of $R$ ). Conversely, given a formal group law $F$, it determines a formal group $\mathcal{F}=\operatorname{Spf} R \llbracket x_{F} \rrbracket$ in a similar way.

2.2 Subgroups and isogenies By (finite) subgroups of a formal group over $R$, we mean finite flat closed subgroup schemes. Their points are often defined over an extension $\widetilde{R}$ of $R$.

An isogeny $\psi: \mathcal{G} \rightarrow \mathcal{G}^{\prime}$ over $R$ is a finite flat morphism of formal groups. Along $\psi^{*}$, $\mathcal{O}_{G}$ becomes a free $\mathcal{O}_{G^{\prime}}$-module of finite rank $d$, called the degree of $\psi$. Since the residue characteristic of $R$ is $p, d$ must be a power of $p$. 
Suppose $x$ and $x^{\prime}$ are coordinates on $\mathcal{G}$ and $\mathcal{G}^{\prime}$. Then $\psi$ induces a homomorphism $G_{x} \rightarrow G_{x^{\prime}}^{\prime}$ of formal group laws, i.e., $h(t) \in t \cdot R \llbracket t \rrbracket$ such that

$$
h\left(t_{1} \underset{G_{x}}{+} t_{2}\right)=h\left(t_{1}\right) \underset{G_{x^{\prime}}^{\prime}}{+} h\left(t_{2}\right)
$$

In fact, $h(x)=\psi^{*}\left(x^{\prime}\right)$ and sometimes we will abuse notation by writing $\psi$ for $h$. We will also denote this homomorphism by $\psi: G \rightarrow G^{\prime}$ and say it is an isogeny of degree $d$ (cf. [Lubin1967, 1.6]). By Weierstrass preparation, $h=m n$ with $m \in R[t]$ monic of degree $d$ and $n \in R \llbracket t \rrbracket$ invertible.

2.3 Kernels and quotients The notions of subgroups and of isogenies are connected as follows.

Given $\psi: \mathcal{G} \rightarrow \mathcal{G}^{\prime}$ as above, its kernel $\mathcal{K}$ is defined by $\mathcal{O}_{K}=\mathcal{O}_{G} \otimes_{\mathcal{O}_{G^{\prime}}} R$, where the tensor product is taken along $\psi^{*}$ and the augmentation map of $\mathcal{O}_{G^{\prime}}$. It is naturally a subgroup of $\mathcal{G}$ and has degree $d$ as an effective Cartier divisor in $\mathcal{G}$.

Conversely, given a subgroup $\mathcal{H} \subset \mathcal{G}$ over $\widetilde{R}$ of degree $p^{r}$, there is a corresponding isogeny $f_{H}: \mathcal{G} \rightarrow \mathcal{G} / \mathcal{H}$ defined by an equalizer diagram

$$
\mathcal{O}_{G / H} \stackrel{f_{H}^{*}}{\longrightarrow} \mathcal{O}_{G} \underset{\pi^{*}}{\stackrel{\mu^{*}}{\longrightarrow}} \mathcal{O}_{G \times H}
$$

where $\mu, \pi: \mathcal{G} \times \mathcal{H} \rightarrow \mathcal{G}$ are the multiplication, projection maps, and $\mathcal{G} / \mathcal{H}$ is naturally a formal group over $\widetilde{R}$. Moreover, given a coordinate $x$ on $\mathcal{G}$,

$$
x_{H}:=\operatorname{Norm}_{f_{H}^{*}}(x)
$$

is a coordinate on $\mathcal{G} / \mathcal{H}$, where $\operatorname{Norm}_{f_{H}^{*}}(x)$ equals the determinant of multiplication by $x$ on $\mathcal{O}_{G}$ as a finite free $\mathcal{O}_{G / H}$-module via $f_{H}^{*}$. Explicitly,

$$
f_{H}^{*}\left(x_{H}\right)=\prod_{Q \in \mathcal{H}(\widetilde{R})}(x+\underset{G}{ } x(Q))
$$

By writing $f_{H}: G \rightarrow G / H$ as an isogeny of formal group laws, we will always intend the above compatibility between corresponding coordinates. Sometimes we write more specifically

$$
f_{H}^{x}: G_{x} \rightarrow G_{x} / H:=(G / H)_{x_{H}}
$$

Note that over the residue field of $R,(2.4)$ becomes

$$
f_{H}^{*}\left(x_{H}\right)=x^{p^{r}}
$$


as a formal group over a field of characteristic $p$ has exactly one subgroup of degree $p^{r}$. Thus $f_{H}$ is a lift of the relative $p^{r}$-power Frobenius isogeny.

For more details, see [Lubin1967, §1, esp. Theorems 1.4, 1.5], [Strickland1997, §5, esp. Theorem 19] (cf. Remark 8.14 below), and [Ando1995, §§2.1-2.2].

2.6 Complex cobordism Let $M U\langle 0\rangle$ be the Thom spectrum of the tautological (virtual) complex vector bundle over $\mathbb{Z} \times B U$. We have $\pi_{*} M U\langle 0\rangle \cong \pi_{*} M U\left[\beta^{ \pm 1}\right]$ with $|\beta|=2$. More generally, let $M U\langle 2 k\rangle$ be the Thom spectrum associated to the $(2 k-1)$-connected cover $B U\langle 2 k\rangle \rightarrow \mathbb{Z} \times B U$.

The spectrum $M U\langle 0\rangle$ is often written $M U P$ or $M P$ for "periodic" (as can be seen from its homotopy groups). In fact,

$$
M U\langle 0\rangle=\bigvee_{m \in \mathbb{Z}} \Sigma^{2 m} M U
$$

so that $\pi_{0} M U\langle 0\rangle$ is the ring of cobordism classes of even-dimensional stably almost complex manifolds. This ring carries the universal formal group law of Lazard by [Quillen1969, Theorem 2].

The spectrum $M U\langle 2\rangle=M U$. The homology of $M U\langle 2 k\rangle$ is concentrated in even degrees if $0 \leq k \leq 3$.

2.7 Morava E-theories Let $k$ be a perfect field of characteristic $p$, and $\mathcal{G}$ be a formal group over $k$ of finite height $n$. Associated to this data, there is a generalized cohomology theory, called a Morava E-theory (of height $n$ at the prime $p$ ). It is represented by a ring spectrum $\boldsymbol{E}=\boldsymbol{E}_{n}$. The formal scheme $\mathcal{G}_{E}:=\operatorname{Spf} \boldsymbol{E}^{0} \mathbb{C P}^{\infty}$ is naturally a formal group over $\boldsymbol{E}^{0}(\mathrm{pt})=\pi_{0} \boldsymbol{E}$. The above association requires that $\mathcal{G}_{\boldsymbol{E}}$ be a Lubin-Tate universal deformation of $\mathcal{G} / k$ (see $\S 4$ below). We have

$$
\pi_{*} \boldsymbol{E} \cong \mathbb{W} k \llbracket u_{1}, \ldots, u_{n-1} \rrbracket\left[u^{ \pm 1}\right]
$$

where $\left|u_{i}\right|=0$ and $|u|=2 .^{1}$

Thus a Morava E-theory spectrum is a topological realization of a Lubin-Tate ring. Strickland showed that $\boldsymbol{E}^{0} B \Sigma_{p^{r}} / I_{\mathrm{tr}}$ is a finite free module over $\pi_{0} \boldsymbol{E}$, where $I_{\mathrm{tr}}$ is the ideal generated by the images of transfers from proper subgroups of the symmetric group $\Sigma_{p^{r}}$ on $p^{r}$ letters. Moreover, this ring classifies degree- $p^{r}$ subgroups of $\mathcal{G}_{E}$ [Strickland1998, Theorem 1.1] (see §5). Ando, Hopkins, and Strickland then assembled these into a topological realization of descent data for level structures on $\mathcal{G}_{E}$ in [Ando-Hopkins-Strickland2004, §3.2] (see §8).

\footnotetext{
${ }^{1}$ For some purposes, it is convenient to instead have $\mathbb{W} \bar{k}$ or $|u|=-2$ in $\pi_{*} \boldsymbol{E}$.
} 
When $\mathcal{G}$ is the formal group of a supersingular elliptic curve, its corresponding Etheory (of height 2) is an elliptic cohomology theory [Ando-Hopkins-Strickland2001, Definition 1.2] via the Serre-Tate theorem.

2.8 Complex orientations for Morava E-theories A complex orientation for $\boldsymbol{E}$ is a coherent choice of Thom class in $\boldsymbol{E}$-cohomology for every complex vector bundle. It amounts to the choice of a single class $\xi \in \widetilde{\boldsymbol{E}}^{2} \mathbb{C P}^{\infty}$ which restricts to 1 under the composite

$$
\widetilde{\boldsymbol{E}}^{2} \mathbb{C P} \mathbb{P}^{\infty} \rightarrow \widetilde{\boldsymbol{E}}^{2} \mathbb{C P}^{1}=\widetilde{\boldsymbol{E}}^{2} S^{2} \cong \boldsymbol{E}^{0}(\mathrm{pt})
$$

Given a coordinate $x \in \mathcal{O}_{G_{E}}=\boldsymbol{E}^{0} \mathbb{C P}^{\infty}$, as a trivialization for $\mathcal{I}_{G_{E}}(O)=\widetilde{\boldsymbol{E}}^{0} \mathbb{C P}^{\infty}$, it corresponds to an invertible class $u_{x} \in \widetilde{\boldsymbol{E}}^{0} \mathbb{C P}^{1} \cong \pi_{2} \boldsymbol{E}$. We then get a complex orientation for $\boldsymbol{E}$ from $x \cdot u_{x}^{-1} \in \widetilde{\boldsymbol{E}}^{2} \mathbb{C P}^{\infty}$. Conversely, we recover a coordinate on $\mathcal{G}_{\boldsymbol{E}}$ from a class $\xi$ above and a generator for $\pi_{2} \boldsymbol{E}$.

An $M U\langle 0\rangle$-orientation for $\boldsymbol{E}$ is a map $M U\langle 0\rangle \rightarrow \boldsymbol{E}$ of homotopy commutative ring spectra. Consider the natural map

$$
\mathbb{C P}_{+}^{\infty} \rightarrow\left(\mathbb{C P}^{\infty}\right)^{\mathcal{L}} \rightarrow \Sigma^{2} M U \rightarrow M U\langle 0\rangle
$$

where $\mathcal{L}$ is the tautological line bundle over $\mathbb{C P}^{\infty}$. Composing with this, each $M U\langle 0\rangle$-orientation gives a generator of $\widetilde{\boldsymbol{E}}^{0} \mathbb{C} \mathbb{P}^{\infty}$ and thus a coordinate on $\mathcal{G}_{\boldsymbol{E}}$. In fact, the correspondence is a bijection (see [Ando2000, Proposition 1.10(ii)] and [Ando-Hopkins-Strickland2001, Corollary 2.50]).

2.9 $\mathbf{E}_{\infty}$ and $\mathbf{H}_{\infty}$ structures Let $\mathrm{Sp}$ be a complete and cocomplete category of spectra, indexed over some universe, with an associative and commutative smash product $\wedge$ (e.g., the category of $\mathbb{L}$-spectra in [Elmendorf-Kriz-Mandell-May1997, Chapter I]).

An $E_{\infty}$-ring spectrum is a commutative monoid in Sp. Equivalently, it is an algebra for the monad $\mathbb{D}$ on $\mathrm{Sp}$ defined by

$$
\mathbb{D}(-):=\bigvee_{m \geq 0} \mathbb{D}_{m}(-):=\bigvee_{m \geq 0}(-)^{\wedge m} / \Sigma_{m}
$$

where $\Sigma_{m}$ is the symmetric group on $m$ letters acting on the $m$-fold smash product.

Weaker than being $\mathrm{E}_{\infty}$, an $H_{\infty}$-ring spectrum is a commutative monoid in the homotopy category of Sp. It also has a description as an algebra for the monad which descends from $\mathbb{D}$ to the homotopy category. In particular, there are power operations $D_{m}$ on the homotopy groups of such a spectrum (see [Bruner-May-McClure-Steinberger1986, Chapter I]). 
Complex cobordism $M U$ and its variants above are $\mathrm{E}_{\infty}$-ring spectra [May1977, § IV.2]. Morava E-theories $\boldsymbol{E}$ are also $\mathrm{E}_{\infty}$-ring spectra [Goerss-Hopkins2004, Corollary 7.6]. A morphism of $\mathrm{E}_{\infty}$-ring (or $\mathrm{H}_{\infty}$-ring) spectra is called an $E_{\infty}$ (or $H_{\infty}$ ) map.

\section{Wide categories of formal groups}

3.1 The category FG and its subcategories Consider FG whose objects are formal groups

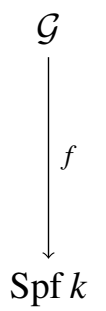

of finite height over variable base fields of characteristic $p$, and whose morphisms are cartesian squares

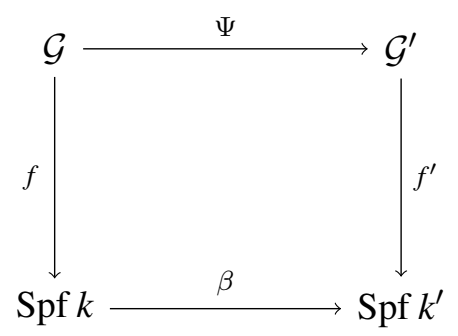

i.e., commutative squares such that the induced morphism of $k$-schemes

$$
\mathcal{G} \stackrel{(\Psi, f)}{\longrightarrow} \mathcal{G}^{\prime} \underset{k^{\prime}}{\times} k
$$

is a homomorphism of formal groups over $k$. We also have subcategories $\mathrm{FG}_{\text {isog }}$ and $\mathrm{FG}_{\text {iso }}$ when (3.3) is restricted to be an isogeny or isomorphism. Write $\mathrm{FG}(k)$, $\mathrm{FG}_{\text {isog }}(k)$, and $\mathrm{FG}_{\text {iso }}(k)$ for the subcategories where the base field is fixed and $\beta=\mathrm{id}$ in (3.2). 
We think of $\mathrm{FG}, \mathrm{FG}_{\text {isog }}, \mathrm{FG}_{\text {iso }}$ as "wide" categories given the factorization

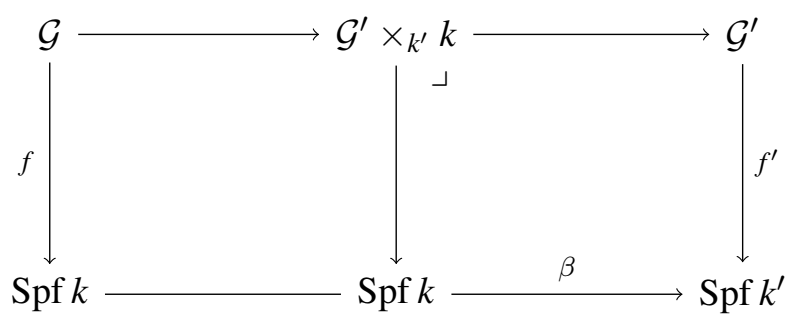

Example 3.5 For our purpose, a key example of morphisms in FG is the following, where $\sigma$ is the absolute $p$-power Frobenius and Frob is the relative one.

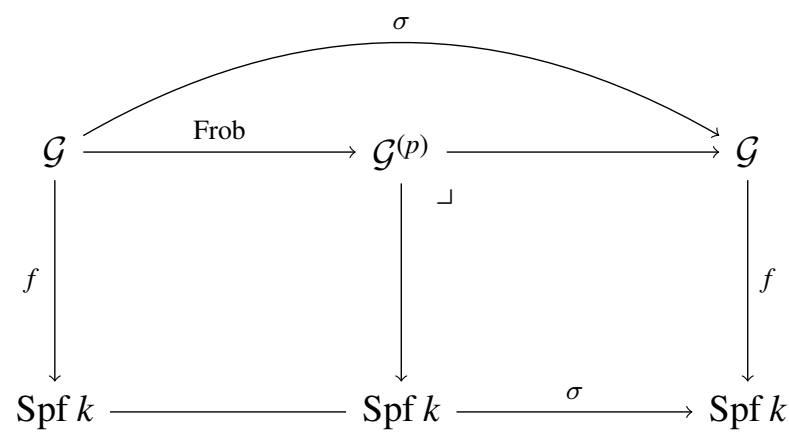

This is an endomorphism in $\mathrm{FG}_{\text {isog }}$ on the object $\mathcal{G} / k$. Denote it by $\Phi$. It is not a morphism in $\mathrm{FG}_{\text {isog }}(k)$. The composite $\Phi^{r}$ corresponds to the $p^{r}$-power Frobenius.

3.7 Canonical factorization of $\Phi^{r}$ along an isogeny Given any $\psi: \mathcal{G} \rightarrow \mathcal{G}^{\prime}$ in $\mathrm{FG}_{\text {isog }}(k)$, necessarily of degree $p^{r}$ for some $r \geq 0$, there is a unique factorization in $\mathrm{FG}_{\text {isog }}$ of $\Phi^{r}$ along $\psi$ as follows, where $\Phi^{r}=\Lambda_{\psi} \circ \psi$ with $\Lambda_{\psi}$ in $\mathrm{FG}_{\text {iso }}$.

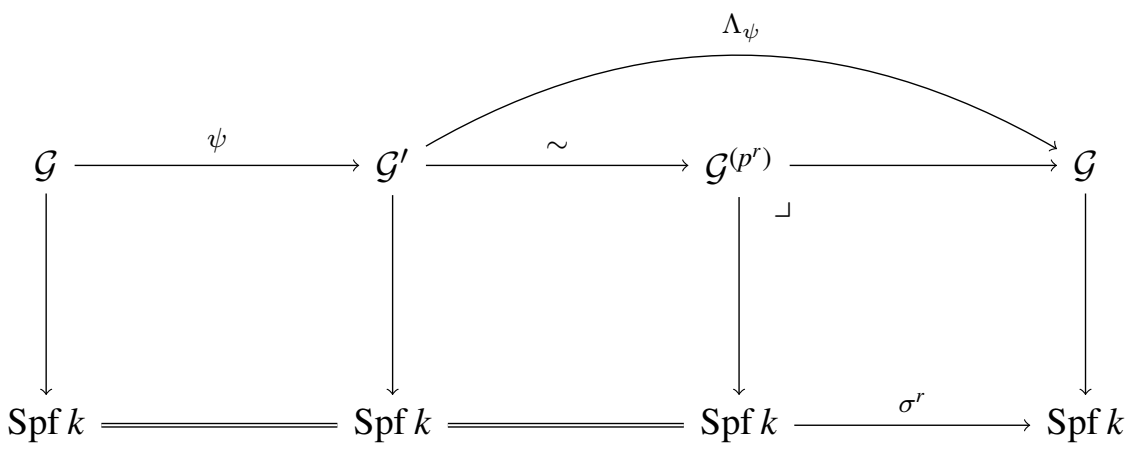


Correspondingly, between rings of functions on the formal groups, we have

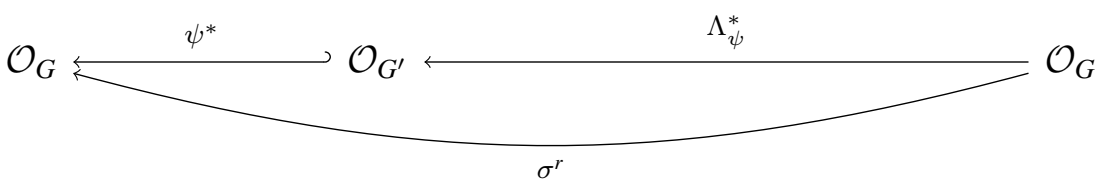

When $\psi$ is the relative Frobenius, the map $\Lambda_{\psi}^{*}$ has a simple description below.

Lemma 3.10 Let $\psi=\mathrm{Frob}^{r}$ in (3.8). Then $\Lambda_{\psi}^{*}$ coincides with the norm of $\mathcal{O}_{G}$ as a finite free module over $\mathcal{O}_{G^{\prime}}$ along $\psi^{*}$; that is, given any $a \in \mathcal{O}_{G}, \Lambda_{\psi}^{*}(a)$ equals the determinant of multiplication by $a$ as an $\mathcal{O}_{G^{\prime}}$-linear transformation on $\mathcal{O}_{G}$.

Proof Let $\mathcal{O}_{G}=k \llbracket x \rrbracket$ and $\mathcal{O}_{G^{\prime}}=k \llbracket y \rrbracket$ with $\psi^{*}(y)=x^{p^{r}}$. Write the norm map as $\operatorname{Norm}_{\psi^{*}}: \mathcal{O}_{G} \rightarrow \mathcal{O}_{G^{\prime}}$. We have

$$
\operatorname{Norm}_{\psi^{*}}(x)=\prod_{i=1}^{p^{r}} x_{i}=(-1)^{p^{r}+1} y=y
$$

and $\operatorname{Norm}_{\psi^{*}}(c)=c^{p^{r}}$, where $c \in k$ and $x_{i}$ are the roots of the minimal polynomial of $x$ over $\mathcal{O}_{G^{\prime}}$. Note that in characteristic $p$, the norm map is additive and hence a local homomorphism. Thus composing with the $k$-linear map $\psi^{*}$, it becomes the absolute $p^{r}$-power Frobenius $\sigma^{r}$ as follows, where $h^{\left(p^{r}\right)}$ is the series obtained by twisting the coefficients of $h$ with the $p^{r}$-power Frobenius.

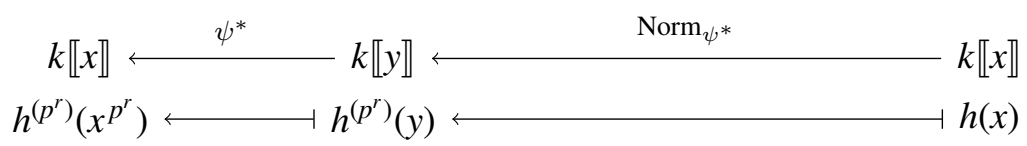

The claim then follows by the uniqueness of the factorization (3.8).

\section{Deformations of formal group laws}

4.1 Set-up Let $k$ be a field of characteristic $p>0$, and $G$ be a formal group law over $k$ of height $n<\infty$. Let $R$ be a complete local ring with maximal ideal $\mathfrak{m}$ and residue field $R / \mathfrak{m} \supset k$, and let $\pi: R \rightarrow R / \mathfrak{m}$ be the natural projection.

4.2 Deformations and deformation structures A deformation of $G$ to $R$ is a triple $(F, i, \eta)$ consisting of a formal group law $F$ over $R$, an inclusion $i: k \hookrightarrow R / \mathfrak{m}$ of fields, and an isomorphism $\eta: \pi^{*} F \stackrel{\sim}{\rightarrow} i^{*} G$ of formal group laws over $R / \mathfrak{m}$, as in 
the following commutative diagram. The leftmost column is supposed to "deform" or "thicken" the rightmost column.

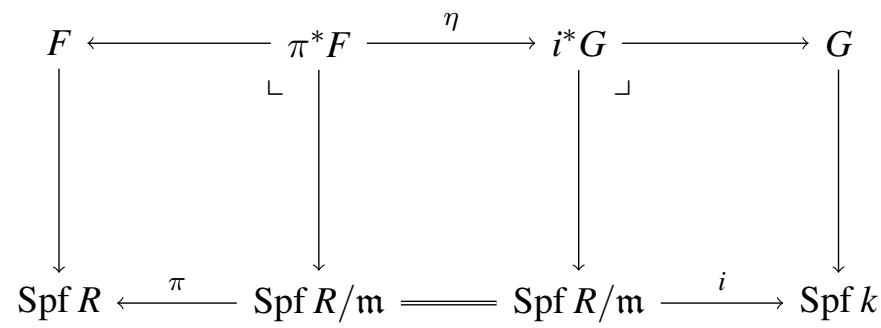

We call the pair $(i, \eta)$ a deformation structure attached to $F$ with respect to $G / k$, and may simply call $F$ a deformation of $G$ to $R$ if its deformation structure is understood. We also call the corresponding formal group $\mathcal{F}$ a deformation of the formal group $\mathcal{G}$ to $R$.

4.3 Base change of deformation structures Let $\beta: R \rightarrow S$ be a local homomorphism, and $\bar{\beta}: R / \mathfrak{m} \rightarrow S / \mathfrak{n}$ be the induced map between residue fields. Given a deformation $(F, i, \eta)$ of $G$ to $R$, there is a deformation $\left(\beta^{*} F, i^{\prime}, \eta\right)$ to $S$ by base change along $\beta$ such that the following diagram commutes. We write $\beta^{*}(i, \eta):=\left(i^{\prime}, \eta\right)=(\bar{\beta} \circ i, \eta)$.

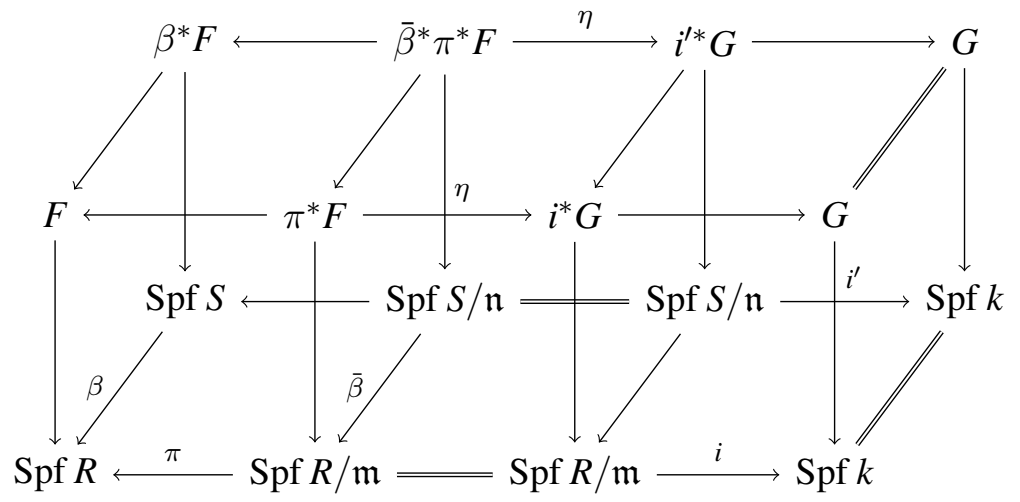

4.4 $\star$-isomorphisms Let $(F, i, \eta)$ and $\left(F^{\prime}, i^{\prime}, \eta^{\prime}\right)$ be deformations of $G / k$ to $R$. A $\star$-isomorphism $(F, i, \eta) \rightarrow\left(F^{\prime}, i^{\prime}, \eta^{\prime}\right)$ consists of an equality $i=i^{\prime}$ and an isomorphism $\psi: F \stackrel{\sim}{\rightarrow} F^{\prime}$ of formal group laws over $R$ such that $\eta^{\prime} \circ \pi^{*} \psi=\eta$, as in the following 
commutative diagram.

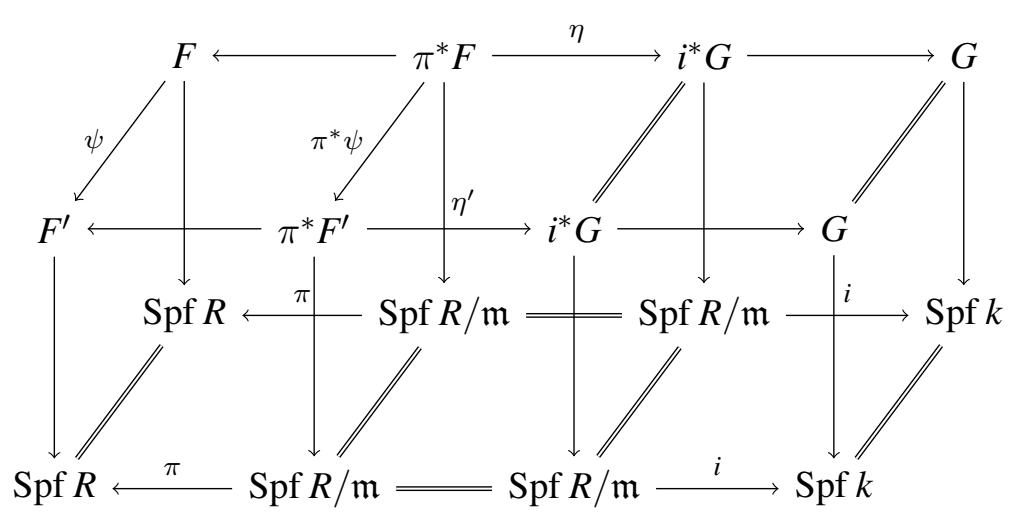

Continuing with the above definition, we simply call $\psi: F \rightarrow F^{\prime}$ a $\star$-isomorphism if in addition $\eta=\eta^{\prime}$ so that $\pi^{*} \psi=$ id. We use the symbol $\stackrel{\star}{=}$ for this equivalence relation. Clearly it is preserved under base change.

Proposition 4.6 (cf. [Rezk2014, Proposition 4.2]) Let $k, G, R$ be as in (4.1) and fix $G / k$. Then the functor

$$
R \mapsto\{\star \text {-isomorphism classes of deformations }(F, i, \eta) \text { of } G \text { to } R\}
$$

from the category of complete local rings with residue field containing $k$ to the category of sets is co-represented by the ring $E_{n}:=\mathbb{W} k \llbracket u_{1}, \ldots, u_{n-1} \rrbracket$. Explicitly, there is a (by no means unique $)^{2}$ deformation $\left(F_{\text {univ }}, \mathrm{id}, \mathrm{id}\right)$ to $E_{n}$ satisfying the following universal property. Given any deformation $(F, i, \eta)$ of $G$ to $R$, there is a unique local homomorphism

$$
\alpha: E_{n} \rightarrow R
$$

such that it reduces to $i: k=E_{n} / I \hookrightarrow R / \mathfrak{m}$, with $I$ and $\mathfrak{m}$ the maximal ideals, and such that there is a unique $\star$-isomorphism

$$
(F, i, \eta) \rightarrow\left(\alpha^{*} F_{\text {univ }}, i, \text { id }\right)
$$

Proof Let $\pi: R \rightarrow R / \mathfrak{m}$ and $\rho: E_{n} \rightarrow E_{n} / I$ be the natural projections. When $\eta$ is allowed to be the identity only, this is [Lubin-Tate1966, Theorem 3.1] (cf. [Ando1995, Theorem 2.3.1]). More generally, the universal property claims that the following

\footnotetext{
${ }^{2}$ See (4.10) below.
} 
diagram commutes, where $\alpha$ and $\psi$ are both unique.

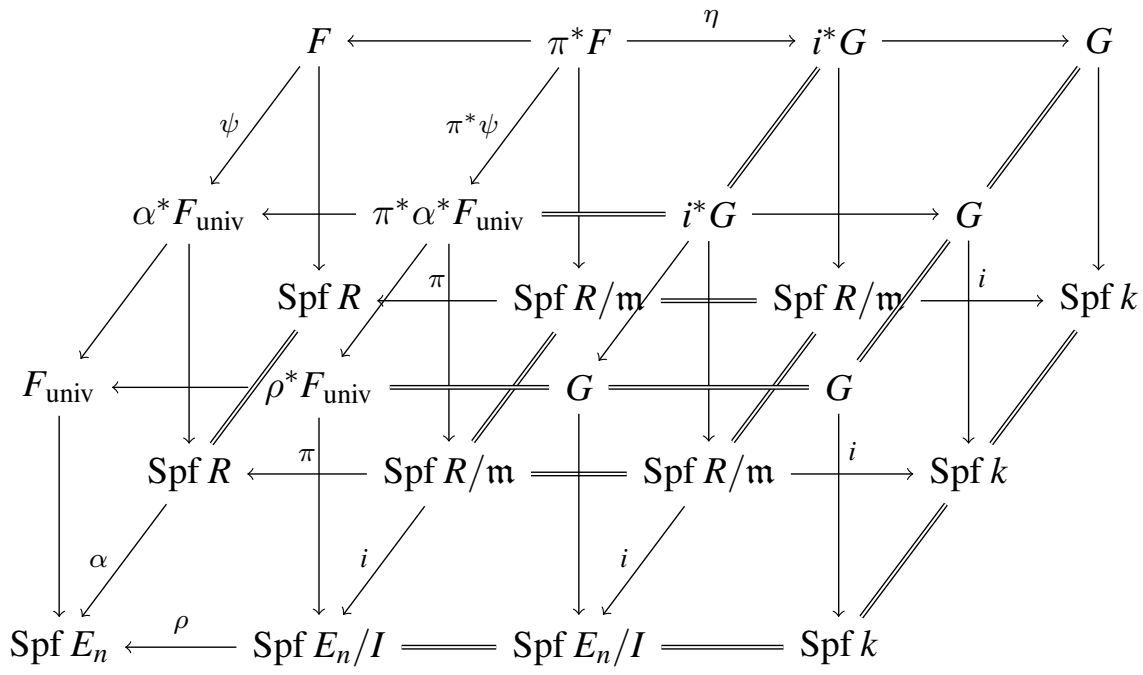

To show this, we refine a half of the diagram as follows, omitting the other half.

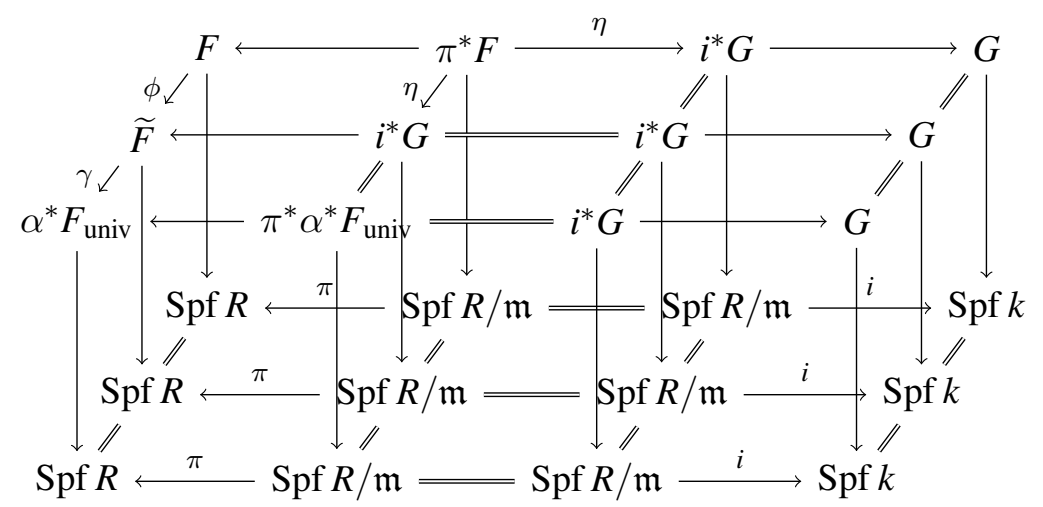

Here $\phi: F \rightarrow \widetilde{F}$ over $R$ is any isomorphism lifting $\eta,{ }^{3}$ so that $\widetilde{F}$ has deformation structure $(i$, id). By [Lubin-Tate1966, Theorem 3.1], there is a unique local homomorphism $\alpha: E_{n} \rightarrow R$ such that it reduces to $i: k=E_{n} / I \hookrightarrow R / \mathfrak{m}$ and such that there is a unique $\star$-isomorphism $\gamma: \widetilde{F} \rightarrow \alpha^{*} F_{\text {univ }}$. Thus (4.9) commutes and, consequently, so does (4.8) if we take $\psi=\gamma \circ \phi$.

Now, to show the uniqueness, suppose that $\alpha^{\prime}$ and $\psi^{\prime}$ fit into (4.8) in place of $\alpha$ and

\footnotetext{
${ }^{3}$ Such lifts always exist because the ring co-representing (strict) isomorphisms between formal group laws over commutative rings is free polynomial. They are in fact unique by the uniqueness in [Lubin-Tate1966, Theorem 3.1].
} 
$\psi$. Then $\alpha^{*} F_{\text {univ }}$ and $\alpha^{\prime *} F_{\text {univ }}$ are in the same $\star$-isomorphism class via $\psi^{\prime} \circ \psi^{-1}$, so by the uniqueness loc. cit. we have $\alpha=\alpha^{\prime}$. Moreover, $\psi^{\prime} \circ \phi^{-1}=\gamma$ so that $\psi^{\prime}=\gamma \circ \phi=\psi$.

4.10 Non-uniqueness of $F_{\text {univ }}$ There can be $F_{\text {univ }} / E_{n}$ and $F_{\text {univ }}^{\prime} / E_{n}$, both satisfying the universal property. Namely, there exists a unique $\alpha_{\text {univ }}: E_{n} \rightarrow E_{n}$ with a unique $F_{\text {univ }}^{\prime} \stackrel{\star}{=} \alpha_{\text {univ }}^{*} F_{\text {univ }}$, and there exists a unique $\alpha_{\text {univ }}^{\prime}: E_{n} \rightarrow E_{n}$ with a unique $F_{\text {univ }} \stackrel{\star}{=}$ $\alpha_{\text {univ }}^{\prime *} F_{\text {univ }}^{\prime}$. Moreover, we have $\alpha_{\text {univ }}^{\prime} \circ \alpha_{\text {univ }}=\alpha_{\text {univ }} \circ \alpha_{\text {univ }}^{\prime}=$ id.

Suppose that a priori we know $F_{\text {univ }} \stackrel{\star}{=} F_{\text {univ }}^{\prime}$. Then $\alpha_{\text {univ }}=\alpha_{\text {univ }}^{\prime}=$ id and this $\star$-isomorphism is unique. Thus the classifying maps $\alpha: E_{n} \rightarrow R$ for $F / R$ are independent of the choice between $F_{\text {univ }}$ and $F_{\text {univ }}^{\prime}$.

\section{Deformations of Frobenius}

The flexibility of having an isomorphism $\eta$ in a deformation of a formal group law buys us a notion of pushforward of deformation structures along any isogeny, compatible with Frobenius in a precise way.

5.1 Pushforward of deformation structures along an isogeny Let $(F, i, \eta)$ be a deformation of $G$ to $R$. Let $\psi: F \rightarrow F^{\prime}$ be an isogeny of formal group laws over $R$ of degree $p^{r}$. Then $F^{\prime}$ can be endowed with a deformation structure $\left(i^{\prime}, \eta^{\prime}\right)$ such that the following diagram commutes, where $\sigma$ is the absolute $p$-power Frobenius and Frob is the relative one.

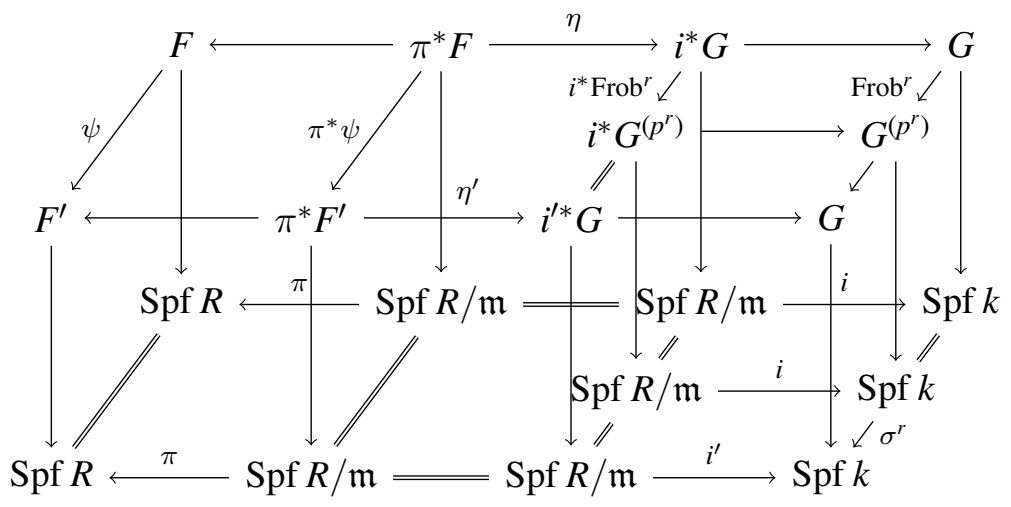

We write $\psi_{!}(i, \eta):=\left(i^{\prime}, \eta^{\prime}\right)$ and call it the pushforward of $(i, \eta)$ along $\psi$. Explicitly, the pair is determined by the equalities

$$
i^{\prime}=i \circ \sigma^{r} \quad \text { and } \quad \eta^{\prime} \circ \pi^{*} \psi=i^{*} \operatorname{Frob}^{r} \circ \eta
$$


5.3 Categories of deformations Fix $G / k$. Let $\operatorname{Def}_{\text {isog }}(R)$ be the category with objects deformations $(F, i, \eta)$ of $G$ to $R$ and with morphisms $(F, i, \eta) \rightarrow\left(F^{\prime}, i^{\prime}, \eta^{\prime}\right)$, each consisting of an isogeny $\psi: F \rightarrow F^{\prime}$ of formal group laws over $R$ and an equality $\left(i^{\prime}, \eta^{\prime}\right)=\psi_{!}(i, \eta)$. The degree of $\psi$ must be $p^{r}$ for some $r \geq 0$. Note that the isomorphisms in $\operatorname{Def}_{\text {isog }}(R)$ are precisely the $\star$-isomorphisms (cf. (4.5), when $r=0$ ) and that the only automorphism of an object is the identity by the uniqueness in Proposition 4.6.

5.4 Deformations of Frobenius Given the diagram (5.2), we view a morphism $(F, i, \eta) \rightarrow\left(F^{\prime}, i^{\prime}, \eta^{\prime}\right)$ in $\operatorname{Def}_{\text {isog }}(R)$ as a deformation to $R$ of $\Phi^{r}$ in the wide category $\mathrm{FG}_{\text {isog }}(3.5) .{ }^{4}$ Thus, we call it a deformation of Frobenius, and simply call $\psi: F \rightarrow F^{\prime}$ such if $\eta=\eta^{\prime}$ so that $\pi^{*} \psi$ is a relative Frobenius (cf. [Rezk2009, 11.3]). Two deformations $\left(F_{1}, i_{1}, \eta_{1}\right) \rightarrow\left(F_{1}^{\prime}, i_{1}^{\prime}, \eta_{1}^{\prime}\right)$ and $\left(F_{2}, i_{2}, \eta_{2}\right) \rightarrow\left(F_{2}^{\prime}, i_{2}^{\prime}, \eta_{2}^{\prime}\right)$ of Frobenius are isomorphic if $\left(F_{1}, i_{1}, \eta_{1}\right)$ and $\left(F_{2}, i_{2}, \eta_{2}\right)$ are $\star$-isomorphic and if $\left(F_{1}^{\prime}, i_{1}^{\prime}, \eta_{1}^{\prime}\right)$ and $\left(F_{2}^{\prime}, i_{2}^{\prime}, \eta_{2}^{\prime}\right)$ are $\star$-isomorphic.

Proposition 5.5 (cf. [Rezk2014, Theorem 4.4]) Let $k, G, R, E_{n}$ be as in Proposition 4.6 and again fix $G / k$. Then, for each $r \geq 0$, the functor

$$
R \mapsto\left\{\text { isomorphism classes of deformations }(F, i, \eta) \rightarrow\left(F^{\prime}, i^{\prime}, \eta^{\prime}\right) \text { of } \Phi^{r} \text { to } R\right\}
$$

from the category of complete local rings with residue field containing $k$ to the category of sets is co-represented by a ring $A_{r}$, which is a bimodule over $A_{0}=E_{n}$ with structure maps local homomorphisms $s_{r}, t_{r}: A_{0} \rightarrow A_{r}$. Explicitly, there is a (by no means unique) deformation ( $F_{\text {univ }}$, id, id) of $G$ to $A_{0}$ satisfying the following universal property. Given any deformation $(F, i, \eta) \rightarrow\left(F^{\prime}, i^{\prime}, \eta^{\prime}\right)$ of $\Phi^{r}$ to $R$, there is a unique local homomorphism

$$
\alpha_{r}: A_{r} \rightarrow R
$$

such that $\alpha_{r} s_{r}, \alpha_{r} t_{r}: A_{0} \rightarrow R$ reduce to $i, i^{\prime}: k=A_{0} / I \hookrightarrow R / \mathfrak{m}$ respectively, with $I$ and $\mathfrak{m}$ the maximal ideals, and such that there are unique $\star$-isomorphisms

$$
(F, i, \eta) \rightarrow\left(\alpha_{r}^{*} s_{r}^{*} F_{\text {univ }}, i, \text { id }\right) \quad \text { and } \quad\left(F^{\prime}, i^{\prime}, \eta^{\prime}\right) \rightarrow\left(\alpha_{r}^{*} t_{r}^{*} F_{\text {univ }}, i^{\prime}, \text { id }\right)
$$

Proof Let $\left(F_{\text {univ }}\right.$, id, id) be given from Proposition 4.6 and write $\mathcal{F}_{\text {univ }}$ for the formal group over $E_{n}$ whose group law is $F_{\text {univ }}$ as in (2.1). Clearly $A_{0}=E_{n}$ with $s_{0}=t_{0}=$ id. In general, for each $r \geq 0$, let $\operatorname{Sub}_{r}\left(\mathcal{F}_{\text {univ }}\right)$ be the affine formal scheme over $A_{0}$ which

\footnotetext{
${ }^{4}$ More precisely, with a corresponding wide category of formal group laws understood, it is a deformation of the endomorphism on $G / k$ induced by $\Phi^{r}$. We will also denote this endomorphism of formal group laws by $\Phi^{r}$.
} 
classifies degree- $p^{r}$ subgroups of $\mathcal{F}_{\text {univ }}$ [Strickland1997, Theorem 42] and let $A_{r}$ be its ring of functions. We need only determine the maps $s_{r}, t_{r}$ and show that $\left(F_{\text {univ }}\right.$, id, id) satisfies the stronger universal property involving $A_{r}$ as stated.

The structure morphism $A_{0} \rightarrow A_{r}$ of $\operatorname{Sub}_{r}\left(\mathcal{F}_{\text {univ }}\right) / A_{0}$ reduces to the identity between residue fields (see [ibid., §13]). Thus $F_{\text {univ }} \times_{A_{0}} A_{r}$ inherits the deformation structure (id, id) from $F_{\text {univ }}$ along the base change. Let $\mathcal{H}_{\text {univ }}^{\left(p^{r}\right)} \subset \mathcal{F}_{\text {univ }} \times_{A_{0}} A_{r}$ be the subgroup of degree $p^{r}$ classified by id: $A_{r} \rightarrow A_{r}$, and let $\mathcal{F}_{\text {univ }}^{\left(p^{r}\right)}:=\left(\mathcal{F}_{\text {univ }} \times_{A_{0}} A_{r}\right) / \mathcal{H}_{\text {univ }}$ be the quotient group as in (2.3) with a particular group law $F_{\text {univ }}^{\left(p^{r}\right)}$. The quotient map of formal groups induces an isogeny

$$
\psi_{\text {univ }}^{\left(p^{r}\right)}: F_{\text {univ }} \underset{A_{0}}{\times} A_{r} \rightarrow F_{\text {univ }}^{\left(p^{r}\right)}
$$

of group laws over $A_{r}$. By (2.5) it is a deformation of Frobenius (5.4) and we have

$$
\psi_{\text {univ! }}^{\left(p^{r}\right)}(\mathrm{id}, \mathrm{id})=\left(\sigma^{r}, \mathrm{id}\right)
$$

In view of Proposition 4.6, let $s_{r}, t_{r}: A_{0} \rightarrow A_{r}$ be the unique local homomorphisms which classify $\left(F_{\text {univ }} \times{ }_{A_{0}} A_{r}\right.$, id, id) and $\left(F_{\text {univ }}^{\left(p^{r}\right)}, \sigma^{r}\right.$,id) respectively. Indeed, by uniqueness, $s_{r}$ is the structure morphism of $\operatorname{Sub}_{r}\left(\mathcal{F}_{\text {univ }}\right) / A_{0}$.

It remains to verify the universal property. By Proposition 4.6, given any deformation $\psi \times \psi_{!}:(F, i, \eta) \rightarrow\left(F^{\prime}, i^{\prime}, \eta^{\prime}\right)$ of $\Phi^{r}$ to $R$, there are unique local homomorphisms

$$
\alpha, \alpha^{\prime}: A_{0} \rightarrow R
$$

such that they reduce to $i, i^{\prime}: k=A_{0} / I \hookrightarrow R / \mathfrak{m}$ respectively, and such that there are unique $\star$-isomorphisms

$$
(F, i, \eta) \rightarrow\left(\alpha^{*} F_{\text {univ }}, i, \text { id }\right) \quad \text { and } \quad\left(F^{\prime}, i^{\prime}, \eta^{\prime}\right) \rightarrow\left(\alpha^{\prime *} F_{\text {univ }}, i^{\prime}, \text { id }\right)
$$

Let $\mathcal{H} \subset \alpha^{*} \mathcal{F}_{\text {univ }}$ be the image of $\operatorname{ker} \psi \subset \mathcal{F}$ under the first $\star$-isomorphism. ${ }^{5}$ It is a subgroup of degree $p^{r}$. Then by [ibid., Theorem 42] (taking $X=\operatorname{Spf} A_{0}$ and $Y=\operatorname{Spf} R$ ) there is a unique local homomorphism

$$
\alpha_{r}: A_{r} \rightarrow R
$$

which classifies $\mathcal{H}$ with $\alpha_{r} \circ s_{r}=\alpha$. Clearly $\alpha_{r} \circ s_{r}$ reduces to $i$ and there is a unique

\footnotetext{
${ }^{5}$ In fact, since a $\star$-isomorphism of formal group laws can be thought of as a change of coordinates on a formal group, we may write $\mathcal{H}=\operatorname{ker} \psi$.
} 
$\star$-isomorphism $(F, i, \eta) \rightarrow\left(\alpha_{r}^{*} s_{r}^{*} F_{\text {univ }}, i\right.$, id $)$ as above. On the other hand, we have

$$
\begin{array}{rlr}
\left(F^{\prime}, i^{\prime}, \eta^{\prime}\right) & \stackrel{\star}{=}\left(F / \operatorname{ker} \psi, i^{\prime}, \eta\right) \\
& \stackrel{\star}{=}\left(\alpha^{*} F_{\text {univ }} / H, i^{\prime}, \text { id }\right) & \\
& =\left(\alpha_{r}^{*}\left(F_{\text {univ }} \times_{A_{0}} A_{r}\right) / \alpha_{r}^{*} H_{\text {univ }}^{\left(p^{r}\right)}, i^{\prime}, \text { id }\right) & \\
& =\left(\alpha_{r}^{*} F_{\text {univ }}^{\left(p^{r}\right)}, i^{\prime}, \text { id }\right) & \text { by [ibid. }, \text { Theorem } 19(\mathrm{v})] \\
& \stackrel{\star}{=}\left(\alpha_{r}^{*} t_{r}^{*} F_{\text {univ }}, i^{\prime}, \text { id }\right) &
\end{array}
$$

Therefore, $\alpha_{r} \circ t_{r}=\alpha^{\prime}$, so $\alpha_{r} \circ t_{r}$ reduces to $i^{\prime}$ and there is a unique $\star$-isomorphism $\left(F^{\prime}, i^{\prime}, \eta^{\prime}\right) \rightarrow\left(\alpha_{r}^{*} t_{r}^{*} F_{\text {univ }}, i^{\prime}\right.$, id $)$.

5.6 Canonical lifts of Frobenius morphisms To summarize Proposition 5.5 and its proof, the ring $A_{r}$ carries a universal example $\psi_{\text {univ }}^{\left(p^{r}\right)}$ of deformation of $\Phi^{r}$ to $R$ as follows. ${ }^{6}$

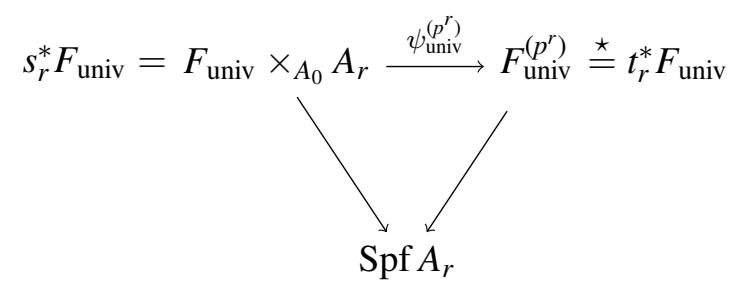

The central notion of norm coherence in this paper, introduced in the next section, concerns the question of when the $\star$-isomorphism in the above diagram is the identity. We write $\psi_{\text {univ }}^{\left(p^{r}\right) \star}$ for the composite of $\psi_{\text {univ }}^{\left(p^{r}\right)}$ with this $\star$-isomorphism.

Remark 5.8 Continuing with (4.10), we see from the proof of Proposition 5.5 that the maps $\alpha_{r}, s_{r}, t_{r}$ are independent of the choice between $\star$-isomorphic universal deformations.

5.9 Dependency of $F_{\text {univ }}$ on $G / k$ The choice of $F_{\text {univ }}=F_{\text {univ }}(G)$ as in $(4.10,5.8)$ can be made functorial with respect to morphisms in $\mathrm{FG}_{\text {isog }}$. Specifically, for functoriality under base change, the right square in (3.4) deforms so that

$$
F_{\text {univ }}\left(G^{\prime} \times_{k^{\prime}} k\right)=F_{\text {univ }}\left(G^{\prime}\right) \underset{E_{n}\left(G^{\prime}\right)}{\times} E_{n}\left(G^{\prime} \times_{k^{\prime}} k\right)
$$

as formal group laws, where $n$ is the height of $G^{\prime} / k^{\prime}$ (invariant under base change) and $E_{n}\left(G^{\prime}\right) \rightarrow E_{n}\left(G^{\prime} \times k^{\prime} k\right)$ sends each generator of the source to the corresponding one

\footnotetext{
${ }^{6}$ See $\left[\right.$ ibid., $\S 10, \S 13$ ] for more about the rings $A_{r}$. For an explicit example, see [Zhu2015b,
} Theorem 1.2] where $r=1$ and $G$ is of height 2 over $k=\overline{\mathbb{F}}_{p}$. 
of the target. This identity follows from the construction of $F_{\text {univ }}$ in [Lubin-Tate1966, Proposition 1.1] as a "generic group law" $\Gamma$.

Moving to the left square of (3.4), let $\psi: \mathcal{G} \rightarrow \mathcal{G}^{\prime} \times_{k^{\prime}} k$ be any isogeny of degree $p^{r}$ and consider the following (cf. (3.8) and (5.2)).

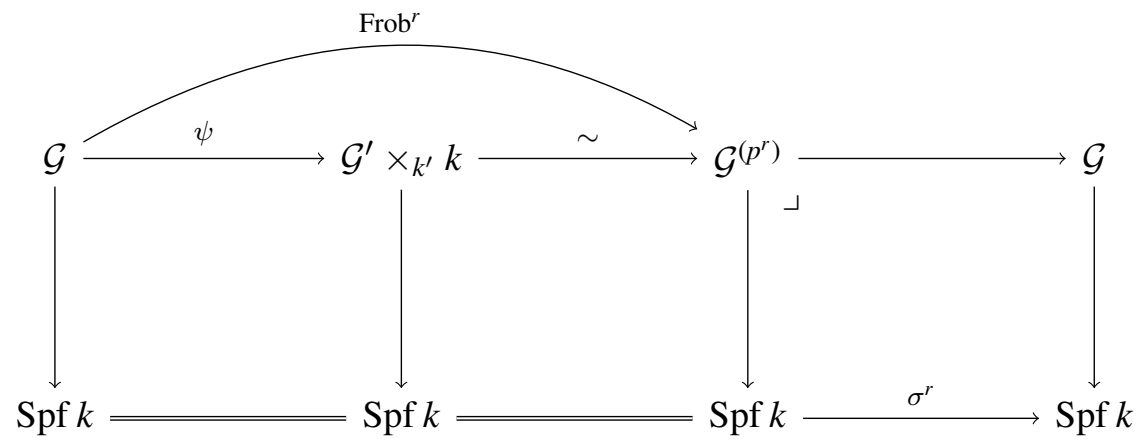

Note that $\operatorname{Frob}^{r}$ deforms to $\psi_{\text {univ }}^{\left(p^{r}\right) \star}: s_{r}^{*} F_{\text {univ }}(G) \rightarrow t_{r}^{*} F_{\text {univ }}(G)$ over $A_{r}$ as in (5.7). Moreover,

$$
\begin{aligned}
t_{r}^{*} F_{\text {univ }}(G) & =F_{\text {univ }}(G) \underset{A_{0}}{\times t_{r}} A_{r} \\
& =F_{\text {univ }}(G) \underset{E_{n}(G)}{\times} E_{n}\left(G^{\left(p^{r}\right)}\right) \underset{E_{n}\left(G^{\left(p^{r}\right)}\right)}{\times} A_{r} \\
& =F_{\text {univ }}\left(G^{\left(p^{r}\right)}\right) \underset{E_{n}\left(G^{\left(p^{r}\right)}\right)}{\times} A_{r}
\end{aligned}
$$

where $E_{n}\left(G^{\left(p^{r}\right)}\right)=\mathbb{W} k \llbracket v_{1}, \ldots, v_{n-1} \rrbracket$ with each $v_{i} \mapsto t_{r}\left(u_{i}\right) \in A_{r} .^{7}$ The construction of $F_{\text {univ }}$ clearly respects isomorphisms so that

$$
F_{\text {univ }}\left(G^{\prime} \times_{k^{\prime}} k\right) \underset{E_{n}\left(G^{\prime} \times k^{\prime}\right.}{\times} E_{n}\left(G^{\left(p^{r}\right)}\right) \cong F_{\text {univ }}\left(G^{\left(p^{r}\right)}\right)
$$

Thus, over $A_{r}$ (omitting the base changes), $\psi$ deforms to

$$
F_{\text {univ }}(G) \stackrel{\psi_{\text {univ }}^{\left(p^{r}\right) \star}}{\longrightarrow} F_{\text {univ }}\left(G^{\left(p^{r}\right)}\right) \stackrel{\sim}{\rightarrow} F_{\text {univ }}\left(G^{\prime} \times k^{\prime} k\right)
$$

This shows the functoriality of $F_{\text {univ }}$ under isogenies.

To summarize, given a morphism in $\mathrm{FG}_{\text {isog }}$ as above, the universal deformations of its source and target can be chosen so that in terms of formal group laws (3.4) deforms

\footnotetext{
${ }^{7}$ For an explicit example, see [Zhu2015b, Theorem 1.6(ii)] where $r=1$ and $G$ is of height $n=2$ over $k=\overline{\mathbb{F}}_{p}$, with $u_{1}=h, t_{1}\left(u_{1}\right)=\psi^{p}(h)$.
} 
over $A_{r}$ as follows.

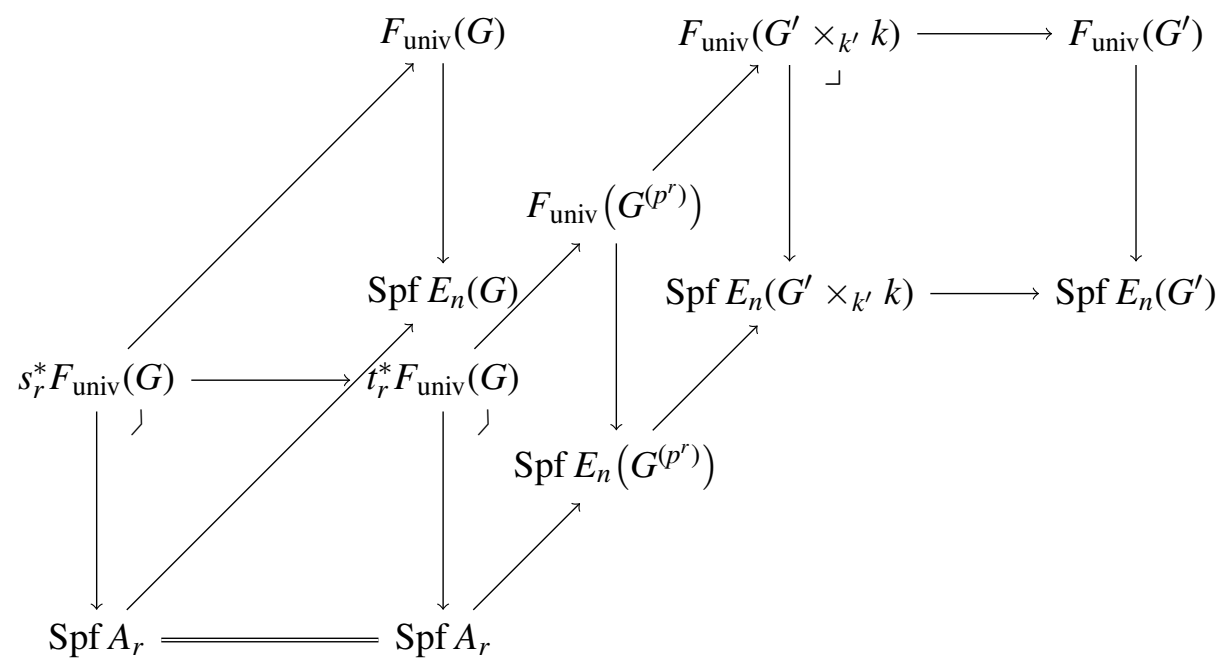

\section{Norm-coherent deformations}

6.1 Set-up Let $k$ be an algebraic extension of $\mathbb{F}_{p}$ (in particular, $k$ is perfect) and $G$ be a formal group law over $k$ of finite height $n$. Let $R$ be a complete local ring with maximal ideal $\mathfrak{m}$ and residue field $R / \mathfrak{m} \supset k$. Let $F / R$ be a deformation of $G / k$ with deformation structure $(i$, id) as in (4.2).

Remark 6.2 Observe that, given any deformation $(F, i, \eta)$, there exists a unique deformation $(\widetilde{F}, i$, id) such that the two are in the same $\star$-isomorphism class (cf. (4.9)). Without loss of generality, here we focus on the case of $\eta=\mathrm{id}$.

6.3 Quotient by the $p$-torsion subgroup As in (2.1) write $\mathcal{F}$ for the formal group over $R$ whose group law is $F$ (upon choosing a coordinate) and write $\mathcal{F}[p]$ for its subgroup scheme of $p$-torsions. This is defined over an extension $\widetilde{R}$ of $R$ obtained by adjoining the roots of the $p$-series of $F$. Let $\mathcal{F} / \mathcal{F}[p]:=\left(\mathcal{F} \times_{R} \widetilde{R}\right) / \mathcal{F}[p]$ be the quotient group as in (2.3) with a particular group law $F / F[p]$ so that the isogeny

$$
f_{p}: F \rightarrow F / F[p]
$$

induced by the quotient morphism of formal groups is a deformation of Frobenius (5.4). Note that $\mathcal{F}[p](\widetilde{R})$ is stable under the action of $\operatorname{Aut}(\widetilde{R} / R)$. Thus $f_{p}$ can be defined over $R$ (cf. [Lubin1967, Theorem 1.4]). 
Remark 6.4 The restriction of $f_{p}$ on the special fiber is the relative $p^{n}$-power Frobenius. It is not an endomorphism unless $k \subset \mathbb{F}_{p^{n}}$ (cf. [Ando1995, proof of Proposition 2.5.1]).

6.5 The isogeny $l_{p} \quad$ By Proposition 5.5 there is a unique local homomorphism $\alpha_{n}: A_{n} \rightarrow R$ together with a unique $\star$-isomorphism $\left(F / F[p], i \circ \sigma^{n}, \mathrm{id}\right) \rightarrow\left(\alpha_{n}^{*} t_{n}^{*} F_{\text {univ }}, i \circ\right.$ $\sigma^{n}$, id). Write

$$
g_{p}: F / F[p] \rightarrow \alpha_{n}^{*} t_{n}^{*} F_{\text {univ }}
$$

for the corresponding $\star$-isomorphism of formal group laws. Let

$$
l_{p}: F \rightarrow \alpha_{n}^{*} t_{n}^{*} F_{\text {univ }}
$$

be the composite $g_{p} \circ f_{p}$.

Remark 6.6 The isogeny $l_{p}$ of formal group laws over $R$ is uniquely characterized by the following properties (cf. [ibid., Proposition 2.5.4], the proof here being completely analogous).

(i) It has source $F$ and target of the form $\alpha^{*} t_{n}^{*} F_{\text {univ }}$ for some local homomorphism $\alpha: A_{n} \rightarrow R$.

(ii) The kernel of $l_{p}$ applied to $\mathcal{F}$ is $\mathcal{F}[p]$.

(iii) Over the residue field, $l_{p}$ reduces to the relative $p^{n}$-power Frobenius.

Explicitly, with notation as in (5.2), $f_{p}$ and $l_{p}$ fit into the following commutative diagram. Their restrictions on the special fiber are highlighted with corresponding decorations, which are in fact identical.

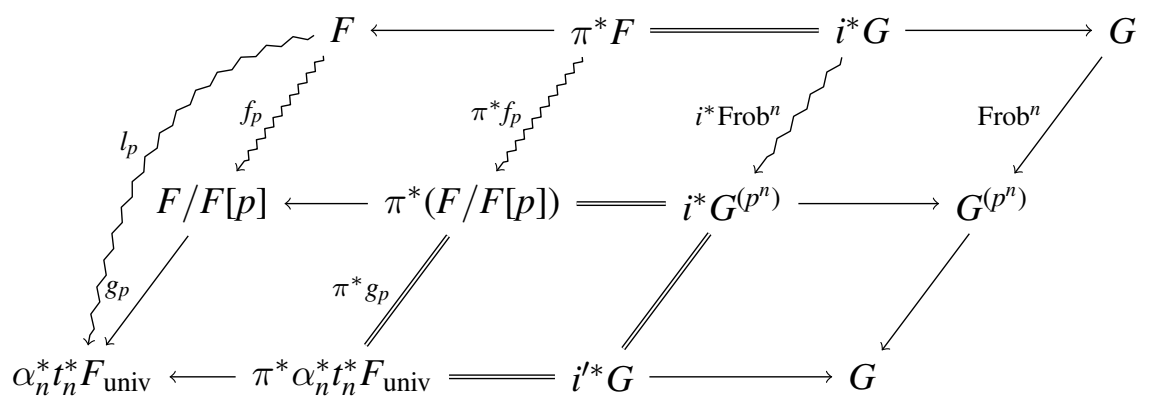

Example 6.8 Let $k=\mathbb{F}_{p}$ and $G$ be the Honda formal group law given by [ibid., 2.5.5] so that $[p]_{G}(t)=t^{p^{n}}$. Then the relative Frobenius Frob $^{n}$ coincides with the absolute Frobenius automorphism on $G$ and so $l_{p}=[p]_{F}$ (cf. [ibid., Proposition 2.6.1]). 
6.9 The isogenies $l_{H}$ More generally, let $\mathcal{H} \subset \mathcal{F}$ be a subgroup of degree $p^{r}$, $\psi_{H}: F \rightarrow F^{\prime}$ be any isogeny with kernel $\mathcal{H}$, and $\psi_{H} \times \psi_{H !}:(F, i$, id $) \rightarrow\left(F^{\prime}, i^{\prime}, \eta^{\prime}\right)$ be the corresponding deformation of Frobenius. The diagram (6.7) generalizes as follows.

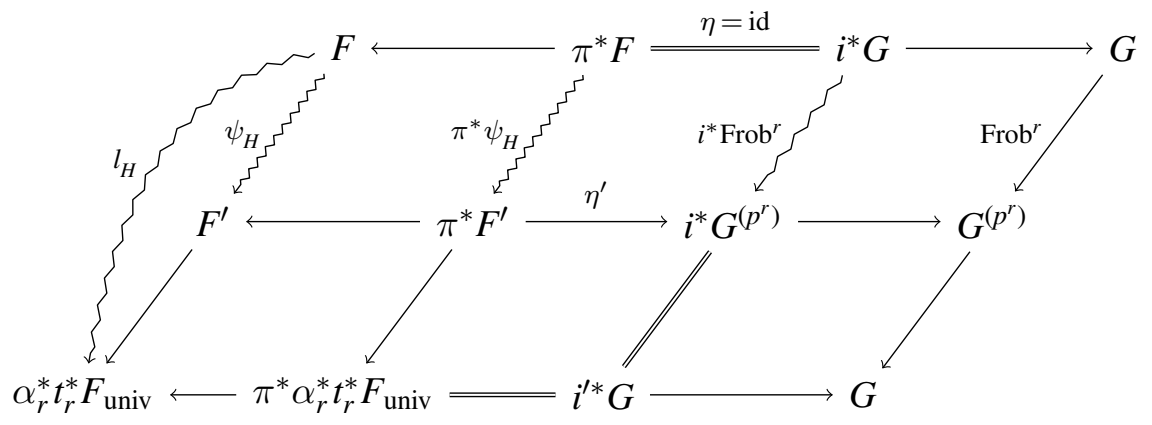

In particular, when $\psi_{H}=f_{H}: F \rightarrow F / H$ is the deformation of Frobenius with kernel $\mathcal{H}$, we have the following commutative diagram.

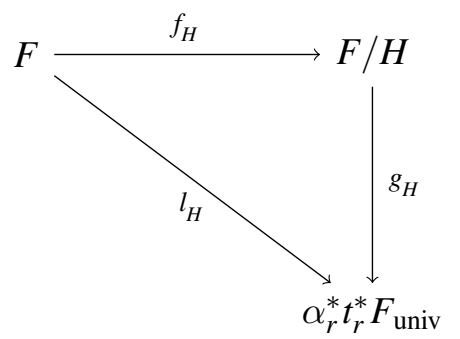

Remark 6.12 This construction of $l_{H}$ is functorial under base change and under quotient, due to the functoriality of $f_{H}$ and $g_{H}$ (see [Strickland1997, Theorem 19(v)], [Ando1995, Proposition 2.2.6], and Proposition 5.5). To be precise, given any local homomorphism $\beta: R \rightarrow R^{\prime}$ and any finite subgroups $\mathcal{H}_{1} \subset \mathcal{H}_{2}$ of $\mathcal{F}$, we have

$$
l_{\beta^{*} H}=\beta^{*} l_{H} \quad \text { and } \quad l_{H_{2} / H_{1}} \circ l_{H_{1}}=l_{H_{2}}
$$

where the composition is taken up to a $\star$-isomorphism, as shown in the following commutative diagrams.

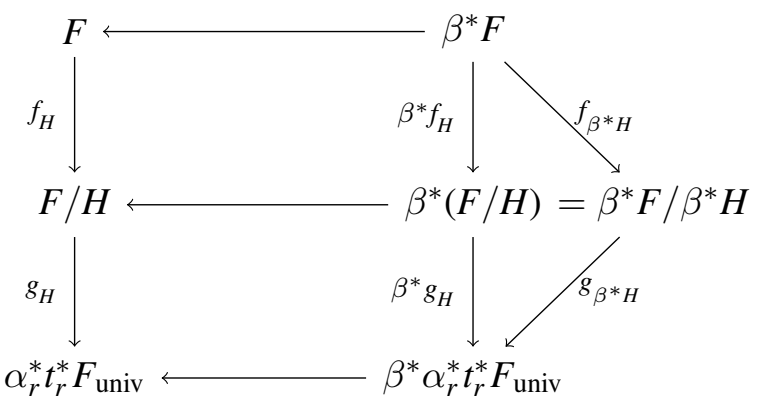




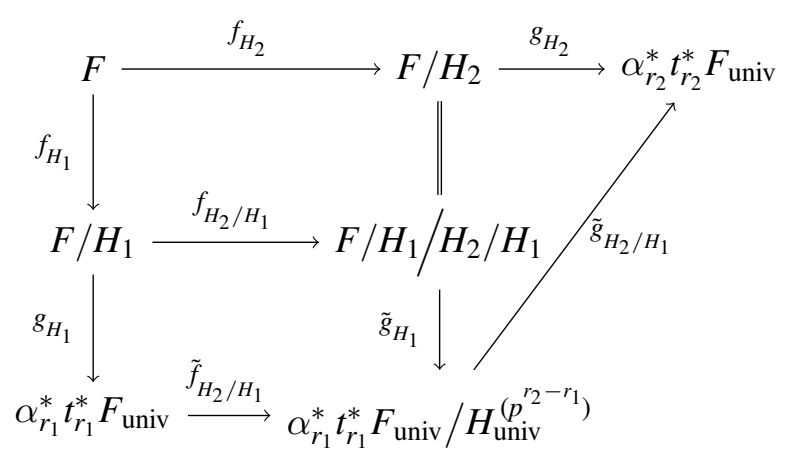

6.15 Definition of norm coherence Recall from the proof of Lemma 3.10 that $\operatorname{Norm}_{\psi^{*}}: \mathcal{O}_{G} \rightarrow \mathcal{O}_{G^{\prime}}$ sends a coordinate $x_{G}$ on $\mathcal{G}$ to the coordinate on $\mathcal{G}^{\prime}=\mathcal{G}^{\left(p^{r}\right)}$ which pulls back along $\psi=\operatorname{Frob}^{r}: \mathcal{G} \rightarrow \mathcal{G}^{\left(p^{r}\right)}$ to $x_{G}^{p^{r}}$. In other words, the norm map agrees with pushing forward a coordinate along the Frobenius isogeny.

This agreement on $x_{G}$ over $k$ may not extend to $R$ for an arbitrary coordinate $x$ on $\mathcal{F}$ lifting $x_{G}$. On one hand, given a subgroup $\mathcal{H} \subset \mathcal{F}$ of degree $p^{r}$, the isogeny $f_{H}: \mathcal{F} \rightarrow \mathcal{F} / \mathcal{H}$ lifts the norm map in the sense that

$$
\begin{aligned}
x_{H}\left(f_{H}(P)\right) & =\prod_{Q \in \mathcal{H}(\widetilde{R})}(x(P)+x(Q)) \\
& =\prod_{\sigma \in \operatorname{Aut}\left(\mathcal{O}_{F} / \mathcal{O}_{F / H}\right)} \sigma \cdot x(P) \\
& =f_{H}^{*} \operatorname{Norm}_{f_{H}^{*}}(x)(P) \\
& =\operatorname{Norm}_{f_{H}^{*}}(x)\left(f_{H}(P)\right)
\end{aligned}
$$

where $x_{H}$ is the coordinate corresponding to the group law $F_{x} / H, P$ is any $R$-point on $\mathcal{F}$, and $\widetilde{R}$ is an extension of $R$ to define the $p^{r}$ points of $\mathcal{H}^{8}$ On the other hand, the

\footnotetext{
${ }^{8} \mathrm{~A}$ detailed proof for the third equality in (6.16) for the norm map, in the context of Galois theory analogous to the situation here, can be found in [Rotman2010, pp. 916-920, esp. Corollary 10.87]. Moreover, consider a coordinate on $\mathcal{F}$ as a map $\mathcal{F} \rightarrow \hat{\mathbb{A}}^{1}(2.1)$. We then have

$$
\mathcal{F} \stackrel{f_{H}}{\longrightarrow} \mathcal{F}^{\prime} \rightarrow \hat{\mathbb{A}}^{1}
$$

and $\operatorname{Norm}_{f_{H}^{*}}$ gives$$
\mathcal{F} \stackrel{x}{\rightarrow} \hat{\mathbb{A}}^{1} \mapsto \quad \mathcal{F}^{\prime} \stackrel{x_{H}}{\longrightarrow} \hat{\mathbb{A}}^{1}
$$

which is analogous to a norm map as a piece of structure in a Tambara functor [Tambara1993, 3.1]. This last notion of a norm map has been packaged into equivariant stable homotopy theory and turned out as a key ingredient in recent advances in the field [Brun2007, Hill-Hopkins2016].
} 
isogeny $l_{H}=g_{H} \circ f_{H}$ lifts Frob $^{r}$ canonically with respect to $\mathcal{H}$; that is, if $f_{H}^{\prime}$ is another lift with kernel $\mathcal{H}$ and classifying $\star$-isomorphism $g_{H}^{\prime}$, then $g_{H}^{\prime} \circ f_{H}^{\prime}=l_{H}$ (Remark 6.6).

Definition 6.17 Let $(F, i$,id) be a deformation of $G$ to $R$ as in (6.1). We say that it is norm-coherent if given any finite subgroup $\mathcal{H}$ of $\mathcal{F}$, the identity

$$
l_{H}=f_{H}
$$

holds. In other words, the condition is that the $\star$-isomorphism $g_{H}=\mathrm{id}$.

More generally, given any deformation $(F, i, \eta)$ of $G$ to $R$, let $(\widetilde{F}, i$, id) be the unique deformation associated to it (Remark 6.2). We say that $(F, i, \eta)$ is norm-coherent if $(\widetilde{F}, i$, id $)$ is.

With the deformation structure understood, we also call the formal group law $F$, as well as its corresponding coordinate $x_{F}$ on $\mathcal{F}$, norm-coherent.

Remark 6.19 Explicitly, in terms of a norm-coherent coordinate $x,(6.18)$ boils down to the identity

$$
h^{\left(p^{r}\right)}\left(l_{H}(x)\right)=\prod_{c \in \mathcal{H}(\widetilde{R})} h\left(x(c){ }_{F} x\right)
$$

for all $h(x) \in R \llbracket x \rrbracket$, where $h^{\left(p^{r}\right)}$ is the series obtained by twisting the coefficients of $h$ with the automorphism on $R$ which lifts the absolute $p^{r}$-power Frobenius (cf. (3.12)). A more conceptual form of this condition is

$$
x_{F^{\prime}, \psi_{!}(i, \eta)}=\operatorname{Norm}_{\psi^{*}}\left(x_{F, i, \eta}\right)
$$

for any deformation $\psi \times \psi$ ! of Frobenius (cf. (6.16)). Indeed, if the isogeny $\psi$ has kernel $\mathcal{H}$, the pushforward $\psi_{!}(i, \eta)$ of deformation structure indicates a change of coordinates on the target so that the left-hand side of (6.20) corresponds to the formal group law $\alpha_{r}^{*} t_{r}^{*} F_{\text {univ }}$ as in (6.10).

6.21 Functoriality of norm coherence Recall from (4.3) and (5.1) the operations of base change and pushforward of deformation structures. The notion of norm coherence in Definition 6.17 is preserved under both as follows.

Proposition 6.22 Let $(F, i, \eta)$ be a norm-coherent deformation of $G$ to $R$.

(i) Given any local homomorphism $\beta: R \rightarrow R^{\prime}$, the deformation $\left(\beta^{*} F, \beta^{*}(i, \eta)\right)$ is norm-coherent. 
(ii) Given any isogeny $\psi: F \rightarrow F^{\prime}$ over $R$, the deformation $\left(F^{\prime}, \psi_{!}(i, \eta)\right)$ is normcoherent. In particular, given any finite subgroup $\mathcal{H} \subset \mathcal{F}$ of degree $p^{r}$, the deformation $\left(F / H, i \circ \sigma^{r}, \eta\right)$ is norm-coherent.

Proof For (i), first note that

$$
\begin{aligned}
& (\widetilde{F}, i, \mathrm{id}) \stackrel{\star}{=}(F, i, \eta) \\
\Longrightarrow & \left(\beta^{*} \widetilde{F}, \beta^{*}(i, \mathrm{id})\right) \stackrel{\star}{=}\left(\beta^{*} F, \beta^{*}(i, \eta)\right) \\
\Longrightarrow & \beta^{*} \widetilde{F}=\widetilde{\beta^{*} F}
\end{aligned}
$$

To see that $\beta^{*} \widetilde{F}$ is norm-coherent, we have from (6.13)

$$
l_{\beta^{*} H}=\beta^{*} l_{H}=\beta^{*} f_{H}=f_{\beta^{*} H}
$$

For (ii), suppose that $\psi$ is of degree $p^{r}$ and let $i^{\prime}=i \circ \sigma^{r}$. In view of

$$
\left(F^{\prime}, \psi_{!}(i, \eta)\right) \stackrel{\star}{=}\left(F / H, i^{\prime}, \eta\right) \stackrel{\star}{=}\left(\widetilde{F / H}, i^{\prime}, \mathrm{id}\right)=\left(\widetilde{F} / H, i^{\prime}, \mathrm{id}\right)
$$

we are reduced to the special case of

$$
(F, i, \text { id }) \stackrel{f_{H} \times f_{H !}}{\longrightarrow}\left(F / H, i \circ \sigma^{r}, \text { id }\right)
$$

Since the source is norm-coherent, we have from (6.14)

$$
l_{H}=f_{H} \quad \text { and } \quad l_{K / H} \circ l_{H}=l_{K}=f_{K}=f_{K / H} \circ f_{H}
$$

where $\mathcal{K}$ is any finite subgroup of $\mathcal{F}$ containing $\mathcal{H}$, and the first composition is onthe-nose because of the first identity in the display. Given that $g_{K / H}$ is an isomorphism, we then deduce from these

$$
l_{K / H}=f_{K / H}
$$

which shows the norm coherence of $\left(F / H, i \circ \sigma^{r}\right.$, id $)$.

\section{Existence and uniqueness of norm-coherent deformations}

The following generalizes a result of Ando's.

Proposition 7.1 (cf. [Ando1995, Theorem 2.5.7]) Let $k, G, R, F$ be as in (6.1) and fix $G / k$. There exists a unique formal group law $F^{\prime}$ over $R$, $\star$-isomorphic to $F$, that is norm-coherent. In other words, given any coordinate $x_{G}$ on the formal group $\mathcal{G}$ and a coordinate $x_{F}$ on $\mathcal{F}$ that lifts $x_{G}$, there exists a unique norm-coherent coordinate on $\mathcal{F}$ whose corresponding formal group law is $\star$-isomorphic to that of $x_{F}$. 
To show this, we will follow Ando's proof of his theorem, making alterations for greater generality whenever necessary (most significantly in (7.6)). The argument breaks into two parts, the first focusing on norm coherence for the $p$-torsion subgroup $\mathcal{F}[p]$ and the second showing functoriality for all finite subgroups. We begin with the following key lemma.

Lemma 7.2 (cf. [ibid., Theorem 2.6.4]) Given any coordinate $x_{F}$ on $\mathcal{F}$ that lifts $x_{G}$, there exists a unique coordinate on $\mathcal{F}$ whose corresponding formal group law is $\star$-isomorphic to that of $x_{F}$ and satisfies

$$
l_{p}=f_{p}
$$

Proof Existence First we reduce the proof to the universal case. Let $F_{\text {univ }}$ be a universal deformation of $G / k$ to $E_{n}$ as in Proposition 4.6, so that there is a unique local homomorphism

$$
\alpha: E_{n} \rightarrow R
$$

together with a unique $\star$-isomorphism

$$
g: F \rightarrow \alpha^{*} F_{\text {univ }}
$$

Suppose that we can construct a coordinate $x$ on $\mathcal{F}_{\text {univ }}$ whose corresponding formal group law $F_{\text {univ }}^{\prime}$ satisfies (7.3) and is $\star$-isomorphic to $F_{\text {univ }}$. Taking $\mathcal{H}=\mathcal{F}_{\text {univ }}[p]$ in the proof of Proposition 6.22(i), we then see that $\alpha^{*} F_{\text {univ }}^{\prime}$ satisfies (7.3) and is $\star$-isomorphic to $F$.

We turn to the universal case. The proof is inductive, on powers of the maximal ideal $I$ of $E_{n}$. Let $y$ be the coordinate corresponding to $F_{\text {univ }}$ from above, so we may write $F_{y}:=F_{\text {univ }}$. With respect to $y$, given that $g_{p}^{y}: F_{y} / F[p] \rightarrow \alpha_{n}^{*} t_{n}^{*} F_{y}$ is defined over $E_{n}$ as in (6.3), let $a(t) \in E_{n} \llbracket t \rrbracket$ be such that

$$
g_{p}^{y}(t)=t+a(t)
$$

We shall construct a desired coordinate $x$ on the universal formal group $\mathcal{F}$ by inductively modifying the coordinate $y$ so that $a(t) \equiv 0 \bmod I^{r}$ for increasing $r$.

Let the inductive hypothesis be

$$
a(t)=\sum_{j \geq 1} a_{j} t^{j} \quad \text { with } \quad a_{j} \in I^{r-1}
$$

Since $g_{p}^{y}$ is a $\star$-isomorphism, we get automatically the case $r=2$. Let $\delta(t)$ be the power series

$$
\delta(t)=t-a^{\left(-p^{n}\right)}(t)
$$


where $a^{\left(-p^{n}\right)}(t)$ is the series obtained by twisting the coefficients $a_{j}$ with the inverse of the local automorphism $\alpha_{n} t_{n}$ on $A_{0}=E_{n}$ and has its coefficients in $I^{r-1}$ as well. ${ }^{9}$ The coordinate

$$
z:=\delta(y)
$$

on $\mathcal{F}$ yields a formal group law $F_{z}$ over $E_{n}$ such that $\delta: F_{y} \rightarrow F_{z}$ is a $\star$-isomorphism. With respect to $z$, let $b(t) \in E_{n} \llbracket t \rrbracket$ be such that

$$
g_{p}^{z}(t)=t+b(t)
$$

We will show that this choice of coordinate $z$ gives

$$
b(t)=\sum_{j \geq 1} b_{j} t^{j} \quad \text { with } \quad b_{j} \in I^{r}
$$

and in particular produces the equation

$$
g_{p}^{z}(t) \equiv t \bmod I^{r}
$$

Note that the formal group laws $F_{y}$ and $F_{z}$ coincide modulo $I^{r-1}$. Thus, by induction and Krull's intersection theorem, we will then obtain in the limit a coordinate $x$ such that $g_{p}^{x}(t)=t$, or $l_{p}^{x}(t)=f_{p}^{x}(t)$, as desired.

Consider the diagram

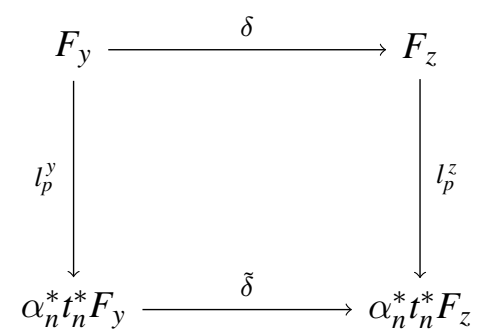

where $\tilde{\delta}:=\alpha_{n}^{*} t_{n}^{*} \delta$ is a $\star$-isomorphism. ${ }^{10}$ By the unique characterization of $l_{p}$ in Remark 6.6, we have $\tilde{\delta} \circ l_{p}^{y} \circ \delta^{-1}=l_{p}^{z}$. Thus the diagram commutes and we get $\tilde{\delta} \circ l_{p}^{y}(t)=l_{p}^{z} \circ \delta(t)$, or

$$
\tilde{\delta} \circ g_{p}^{y} \circ f_{p}^{y}(t)=g_{p}^{z} \circ f_{p}^{z} \circ \delta(t)
$$

\footnotetext{
${ }^{9}$ By Proposition 5.5, $\alpha_{n} t_{n}$ lifts the $p^{n}$-power Frobenius $i^{\prime}=\sigma^{n}$ on $k$ to $\mathbb{W} k$. Moreover, it alters each generator $u_{i}$ of $E_{n}$ by a unit, as any degree- $p^{n}$ isogeny out of $F$ differs by an isomorphism from the multiplication-by- $p$ endomorphism on $F$ (see [Lubin1967, 1.5-1.6]).

${ }^{10}$ The classifying maps for $F_{y} / F[p]$ and $F_{z} / F[p]$ are both $\alpha_{n} t_{n}$ because $F_{y}$ and $F_{z}$ are $\star$-isomorphic (Remark 5.8).
} 
We shall compare the two sides of (7.11) modulo $I^{r}$ to show (7.9) and thus complete the induction.

The left-hand side of (7.11) can be evaluated modulo $I^{r}$ as follows.

$$
\text { (7.12) } \begin{array}{rlr}
\tilde{\delta} \circ g_{p}^{y} \circ f_{p}^{y}(t) & =\tilde{\delta}\left(f_{p}^{y}(t)+a \circ f_{p}^{y}(t)\right) & \text { by (7.4) } \\
& \equiv \tilde{\delta}\left(f_{p}^{y}(t)+a\left(t^{p^{n}}\right)\right) & \text { by (2.5) and (7.5) } \\
& =f_{p}^{y}(t)+a\left(t^{p^{n}}\right)-a\left(f_{p}^{y}(t)+a\left(t^{p^{n}}\right)\right) & \text { by (7.6) } \\
& \equiv f_{p}^{y}(t)+a\left(t^{p^{n}}\right)-a\left(f_{p}^{y}(t)\right) & \text { by (7.5) } \\
& \equiv f_{p}^{y}(t)+a\left(t^{p^{n}}\right)-a\left(t^{p^{n}}\right) & \text { by (2.5) and (7.5) } \\
& =f_{p}^{y}(t) &
\end{array}
$$

For the right-hand side of (7.11), first note that modulo $I^{r}$ we have

$$
\begin{array}{rlr}
f_{p}^{z} \circ \delta(t)= & \text { by (2.4) } \\
= & \prod_{c \in \mathcal{F}[p]\left(\widetilde{E}_{n}\right)}\left(z(c){\underset{F}{z}}_{c} \delta\left(y(c) \underset{F_{y}}{+} t\right)\right. & \text { by (7.7) } \\
= & \prod_{c}\left[\left(y(c) \underset{F_{y}}{c} t\right)-a^{\left(-p^{n}\right)} \underset{F_{y}}{(y(c)+t)]}\right. & \text { by (7.6) } \\
\equiv & \prod_{c}\left(y(c) \underset{F_{y}}{+} t\right) & \\
& -\sum_{c} a^{\left(-p^{n}\right)}\left(y(c) \underset{F_{y}}{+} \prod_{d \neq c}\left(y(d)+{ }_{F_{y}} t\right)\right. & \text { by (7.5) } \\
\equiv & f_{p}^{y}(t)-\sum_{c} a^{\left(-p^{n}\right)}(t) t^{p^{n}-1} & \text { by (2.4), (7.5) and (2.5) } \\
= & f_{p}^{y}(t)-p^{n} a^{\left(-p^{n}\right)}(t) t^{p^{n}-1} & \\
\equiv f_{p}^{y}(t) & \text { by (7.5) and since } p \in I
\end{array}
$$

In particular, by (2.5), this gives

$$
f_{p}^{z} \circ \delta(t) \equiv t^{p^{n}} \bmod I
$$

Thus, given $k \geq 2$, if in (7.8) we have

$$
b(t)=\sum_{j \geq 1} b_{j} t^{j} \quad \text { with } \quad b_{j} \in I^{k-1}
$$

then for $k \leq r$, on the right-hand side of (7.11) we have

$$
\begin{aligned}
g_{p}^{z} \circ f_{p}^{z} \circ \delta(t) & =f_{p}^{z} \circ \delta(t)+b\left(f_{p}^{z} \circ \delta(t)\right) & \text { by (7.8) } \\
& \equiv f_{p}^{y}(t)+b\left(t^{p^{n}}\right) \bmod I^{k} & \text { by (7.13) and (7.14) }
\end{aligned}
$$


Comparing this to (7.12), we get

$$
b(t) \equiv 0 \bmod I^{k}
$$

Since $g_{p}^{z}$ in (7.8) is a $\star$-isomorphism, we can proceed by induction on $k$ and obtain

$$
b(t) \equiv 0 \bmod I^{r}
$$

which implies (7.9).

Uniqueness Let $\mathcal{F} / R$ be a deformation of $\mathcal{G} / k$. Let $x$ and $y$ be two coordinates on $\mathcal{F}$, both lifting $x_{G}$ on $\mathcal{G}$ and both satisfying (7.3). Suppose $F_{x}$ and $F_{y}$ are in the same $\star$-isomorphism class so that there is a $\star$-isomorphism $\delta: F_{x} \rightarrow F_{y}$ fitting into a commutative diagram analogous to (7.10).

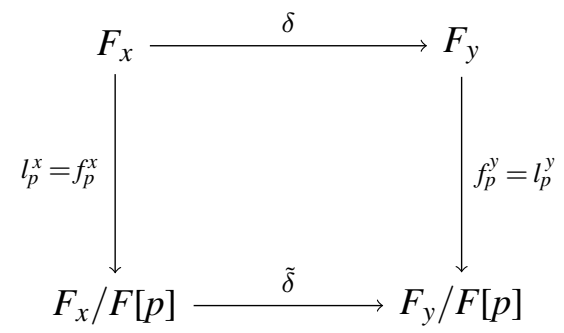

Let $\mathfrak{m}$ be the maximal ideal of $R$. Let $c(t) \in R \llbracket t \rrbracket$ be such that

$$
\delta(t)=t+c(t)
$$

where

$$
c(t)=\sum_{j \geq 1} c_{j} t^{j} \quad \text { with } \quad c_{j} \in \mathfrak{m}
$$

Since $x$ and $y$ are distinct, there exists $r_{0} \geq 2$ such that it is the largest $r$ satisfying

$$
c_{j} \in \mathfrak{m}^{r-1} \quad \text { for all } j
$$

Modulo $\mathfrak{m}^{r_{0}}$ we then have

$$
\begin{aligned}
f_{p}^{y}(t) & =\tilde{\delta} \circ f_{p}^{x} \circ \delta^{-1}(t) \\
& =f_{p}^{x} \circ \delta^{-1}(t)+c^{\left(p^{n}\right)} \circ f_{p}^{x} \circ \delta^{-1}(t) \\
& \equiv f_{p}^{x} \circ \delta^{-1}(t)+c^{\left(p^{n}\right)}\left(t^{p^{n}}\right) \\
& \equiv f_{p}^{y}(t)+c^{\left(p^{n}\right)}\left(t^{p^{n}}\right)
\end{aligned}
$$
analogous to (7.13)

which is a contradiction. 
Proof of Proposition 7.1 (cf. [Ando1995, proof of Proposition 2.6.15]) We need only show that the coordinate $x$ on $\mathcal{F}$ constructed in Lemma 7.2 satisfies the stronger condition $l_{H}=f_{H}$ for any finite $\mathcal{H} \subset \mathcal{F}$. As in the proof of existence there, we are reduced to the universal case with $F_{\text {univ }}=: F=F_{x}$ over $E_{n}$.

Given any $\mathcal{H} \subset \mathcal{F}$ of degree $p^{r}$, we will show that the $\star$-isomorphism

$$
g_{H}: F / H \rightarrow \alpha_{r}^{*} t_{r}^{*} F
$$

is the identity by the uniqueness from Lemma 7.2. Namely, the source and target are in the same $\star$-isomorphism class, and we show that both of them satisfy (7.3). That the target does is clear from the proof of Proposition 6.22 (i). For the source of (7.17), let $p^{-1} \mathcal{H}:=\{c \in \mathcal{F} \mid p c \in \mathcal{H}\} .{ }^{11}$ It contains both $\mathcal{H}$ and $\mathcal{F}[p]$ as subgroups. We need to show

$$
l_{p^{-1} H / H}^{x_{H}}=f_{p^{-1}{ }^{-1} / H}^{x_{H}}
$$

where $x_{H}$ is the coordinate corresponding to the formal group law $F_{x} / H$.

Consider the following commutative diagram, where $x_{p}$ denotes the coordinate corresponding to $F_{x} / F[p]$. The upper rectangle commutes due to the functoriality of the isogeny $f$ under quotient [ibid., Proposition 2.2.6]. The lower rectangle commutes due to the functoriality from Proposition 5.5 of the $\star$-isomorphisms $g$ under quotient. ${ }^{12}$

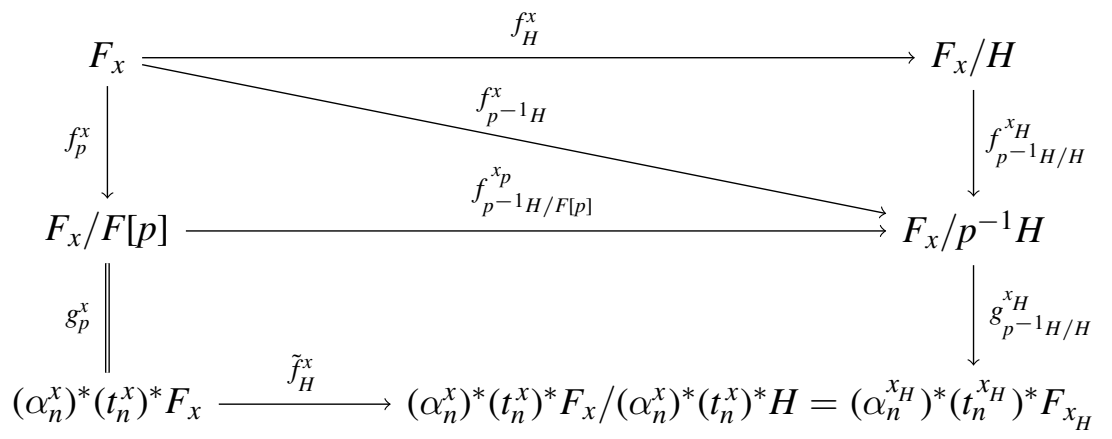

Note that $p^{-1} \mathcal{H} / \mathcal{F}[p] \cong \mathcal{H}$. In the lower rectangle, $g_{p}^{x}=\mathrm{id}$ and hence $f_{p^{-1}{ }_{H / F[p]}}^{x_{p}}=\tilde{f}_{H}^{x}$. This forces the isomorphism $g_{p^{-1} H / H}^{x_{H}}$ to be the identity, and (7.18) follows.

Remark 7.19 In [Zhu2015a, §3.3], for the purpose of studying Hecke operators in elliptic cohomology, we showed the existence of an analogue of Ando's coordinate. It

\footnotetext{
${ }^{11}$ The notation $c \in \mathcal{F}$ means $[c] \subset \mathcal{F}$, where $[c]$ is the effective Cartier divisor defined by a section. To be precise, this set-theoretic description defines the subgroup scheme $p^{-1} \mathcal{H}$ of $\mathcal{F}$ as a sum of effective Cartier divisors.

${ }^{12}$ Here we view $F_{x} / H=F_{x_{H}}$ as a universal deformation with structure $\left(\sigma^{r}\right.$, id) (5.9).
} 
is conceptually different from the norm-coherent coordinates here. Note that there the base change is not along a local homomorphism (cf. [Zhu2014, §4, footnote] and see (7.21) below).

Example 7.20 Let $k=\mathbb{F}_{p^{2}}$ and $G$ be the formal group law of a supersingular elliptic curve over $k$. We choose a curve such that its $p^{2}$-power Frobenius endomorphism coincides with the map of multiplication by $(-1)^{p-1} p$, as in [Zhu2015a, 3.24]. We then have $l_{p}=[-p]$ if $p=2$ by rigidity.

Let $\boldsymbol{E}$ be the Morava E-theory associated to $G / k$, and choose a preferred $\mathcal{P}_{N}$-model for $\boldsymbol{E}$ in the sense of [ibid., Definition 3.29]. In particular, there is a chosen coordinate $u$ on the universal deformation of $\mathcal{G} / k$. Given [ibid., 3.28], the cotangent map along $f_{p}^{u}$ is multiplication by $p$. Thus, by the criterion (7.3), $u$ cannot be norm-coherent if $p=2$.

7.21 More functoriality of norm coherence We continue the discussion in (6.21) with varying $G / k$. Let Coord: $\mathrm{FG}_{\mathrm{isog}} \rightarrow$ Set be the "wide functor"

$$
\mathcal{G} / k \mapsto\{\text { coordinates on } \mathcal{G}\} \subset \mathcal{O}_{G}
$$

in the following sense: given the diagram (3.4), Coord is contravariant along the right square and covariant along the left square. More specifically, Coord is contravariant with respect to base change $\operatorname{Spf} k \rightarrow \operatorname{Spf} k^{\prime}$ and pullback along an isomorphism over $k$, hence contravariant with respect to any morphism in the subcategory $\mathrm{FG}_{\text {iso }}$. On the other hand, given an isogeny $\mathcal{G} \rightarrow \mathcal{G}^{\prime}$ over $k$ of degree $p^{r}$, any coordinate $x$ on $\mathcal{G}$ determines a unique coordinate on $\mathcal{G}^{\left(p^{r}\right)}$ which pulls back along Frob $^{r}$ to $x^{p^{r}}$; this coordinate on $\mathcal{G}^{\left(p^{r}\right)}$ then corresponds to one on $\mathcal{G}^{\prime}$ via the isomorphism between the two formal groups. Thus Coord is also covariant with respect to any morphism in the subcategory $\mathrm{FG}_{\text {isog }}(k)$.

Let $\mathrm{NCoh}: \mathrm{FG}_{\text {isog }} \rightarrow$ Set be the wide functor

$$
\mathcal{G} / k \mapsto\left\{\text { norm-coherent coordinates on } \mathcal{F}_{\text {univ }}(\mathcal{G}) / E_{n}\right\}
$$

where $\mathcal{F}_{\text {univ }}(\mathcal{G})$ is a choice of universal deformation of $\mathcal{G}$ as in (5.9). Its "wideness," in the same sense as above, follows from Proposition 6.22 and the discussion in (5.9).

Theorem 7.22 The natural transformation $\rho:$ NCoh $\rightarrow$ Coord of wide functors by restricting a coordinate on $\mathcal{F}_{\text {univ }}(\mathcal{G})$ to $\mathcal{G}$ is an isomorphism. Moreover, it satisfies Galois descent: given $\mathcal{G} / k$ in $\mathrm{FG}_{\text {isog }}$ and a Galois extension $K / k$, the following 
commutes, where the vertical maps take fixed points under the Galois action.

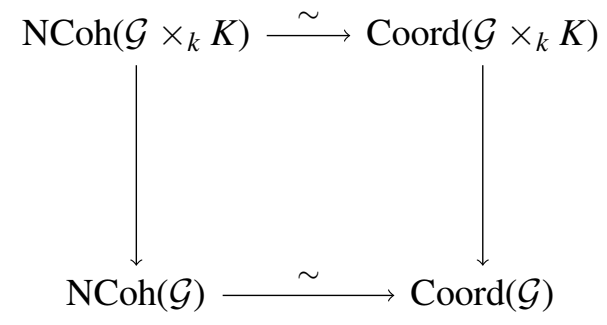

Moreover, this diagram is natural in $\mathcal{G} / k$ and $K / k$.

Proof On each object in $\mathrm{FG}_{\text {isog }}$, the natural transformation $\rho$ is an isomorphism by Proposition 7.1, and the descent is clear since the condition (6.18) of norm coherence is stable under Galois actions. Each of the naturality properties is straightforward to check.

\section{Norm coherence and $\mathbf{H}_{\infty}$ complex orientations}

8.1 Introduction Given a Morava E-theory spectrum $\boldsymbol{E}$, consider its complex orientations, or, more precisely, homotopy multiplicative maps $M U\langle 0\rangle \rightarrow \boldsymbol{E}$. A necessary and sufficient condition for such an orientation to be $\mathrm{H}_{\infty}$ is that its corresponding coordinate on the formal group of $\boldsymbol{E}$ is norm-coherent. Ando showed this for E-theories associated to the Honda formal groups over $\mathbb{F}_{p}$ [Ando1995, Theorem 4.1.1]. There, the norm-coherence condition (6.18) boils down to the identity $[p]=f_{p}$ (cf. (7.3) and (6.8)). Moreover, he established the existence and uniqueness of coordinates, hence orientations, with the desired property [ibid., Theorem 2.6.4].

In fact, to show that norm coherence is necessary and sufficient for $\mathrm{H}_{\infty}$ orientations, Ando's proof does not depend on the choice of the formal groups being the Honda formal groups (see [ibid., Lemma 4.4.4]). However, his setup does require them be defined over $\mathbb{F}_{p}$ so that the relative $p^{r}$-power Frobenius is an endomorphism for every $r \geq 0$ (cf. [ibid., Proposition 2.5.1] and Remark 6.4).

With results in sufficient generality about level structures on formal groups from [Strickland1997], Ando, Hopkins, and Strickland extended the applicability of the above condition for $\mathrm{H}_{\infty}$ orientations: $M U\langle 0\rangle$ generalizes to $M U\langle 2 k\rangle, k \leq 3$, and $\boldsymbol{E}$ generalizes to any even periodic $\mathrm{H}_{\infty}$-ring spectum whose zeroth homotopy is a 
$p$-regular admissible local ring with perfect residue field of characteristic $p$ and whose formal group is of finite height [Ando-Hopkins-Strickland2004, Proposition 6.1]. They did this by first reformulating Ando's condition so that in particular it applies to Etheories associated to formal groups over any perfect field of positive characteristic [ibid., Proposition 4.13].

Based on this general condition, they established the existence and uniqueness of $\mathrm{H}_{\infty} M U\langle 6\rangle$-orientations for $\mathrm{H}_{\infty}$ elliptic spectra, called the sigma orientations, from corresponding norm-coherent cubical structures of elliptic curves [ibid., Proposition 16.5]. However, when the elliptic spectrum represents an E-theory associated to the formal group of a supersingular elliptic curve, such an orientation does not factor through $M U\langle 4\rangle$ due to obstruction from Weil pairings (see [Ando-Strickland2001, proof of Theorem 1.4]). Thus, in this case, we cannot deduce the existence and uniqueness of $\mathrm{H}_{\infty} M U\langle 2 k\rangle$-orientations for $0 \leq k \leq 2$ from the sigma orientation.

8.2 Set-up Let $\boldsymbol{E}$ be a Morava E-theory spectrum, with $\mathcal{G}_{E}=\mathcal{F}_{\text {univ }}(\mathcal{G})$ for some $\mathcal{G} / k$ whose group law is as in (6.1). We will show the existence and uniqueness of $\mathrm{H}_{\infty} M U\langle 0\rangle$-orientations for $\boldsymbol{E}$ by combining Proposition 7.1 with Ando, Hopkins, and Strickland's condition for $\mathrm{H}_{\infty}$ orientations. Specifically, we need only check that their [Ando-Hopkins-Strickland2004, 4.14] and our definition (6.18) for norm coherence agree.

8.3 Descent for level structures on Lubin-Tate formal groups We carry out the needed comparison by recalling the canonical descent data for level structures on $\mathcal{G}_{E}=\mathcal{F}_{\text {univ }}(\mathcal{G})$ from [ibid., Part 3]. Since $\mathcal{G}$ is over $k$ of characteristic $p$, the finite subgroups $\mathcal{H}$ of $\mathcal{G}_{E}$ must be of degree $p^{r}$ for some $r \geq 0$.

Definition 8.4 (cf. [ibid., Definitions 3.1, 9.9, Proposition 10.10 (i), 12.5]) Let $A$ be an "abstract" finite abelian group of order $p^{r}$. Let $S_{\boldsymbol{E}}=\operatorname{Spf} \pi_{0} \boldsymbol{E}$ and $T=\operatorname{Spf} R$ with $R$ as in (6.1). Let $i: T \rightarrow S_{E}$ be a morphism of formal schemes, faithfully flat and locally of finite presentation, which classifies a deformation of $\mathcal{G} / k$ to $R$. Write $\mathcal{A}_{T}$ for the constant formal group scheme of $A$ over $T$. A morphism

$$
\ell: \mathcal{A}_{T} \rightarrow i^{*} \mathcal{G}_{E}
$$

of formal groups over $T$, equivalent to a group homomorphism $\phi_{\ell}: A \rightarrow i^{*} \mathcal{G}_{E}(T)$, is a level $A$-structure on $\mathcal{G}_{E}$ if the effective Cartier divisor $\mathcal{D}_{\ell}:=\sum_{a \in A}\left[\phi_{\ell}(a)\right]$ of degree $p^{r}$ is a subgroup of $i^{*} \mathcal{G}_{E}$.

Remark 8.5 Note that a level $A$-structure $\ell$ on $\mathcal{G}_{E}$ uniquely corresponds to a finite subgroup $\mathcal{H}=\mathcal{D}_{\ell}$, which is different from the scheme-theoretic image of $\mathcal{A}_{T}$ under $\ell$ 
(the latter automatically a subgroup, but possibly of smaller degree). Automorphisms of $A$ correspond to automorphisms of $\mathcal{H}$ (cf. [ibid., Definition 3.1 (3)]).

Definition 8.6 (cf. [ibid., Definition 3.9, Remark 3.12]) Let $\ell: \mathcal{A}_{\operatorname{Spf} R} \rightarrow i^{*} \mathcal{G}_{E}$ be a level $A$-structure on $\mathcal{G}_{E}$ as above. Define $\psi_{\ell}^{E}: \pi_{0} \boldsymbol{E} \rightarrow R$ to be the composite

$$
\pi_{0} \boldsymbol{E} \stackrel{D_{p^{r}}}{\longrightarrow} \pi_{0} \boldsymbol{E}^{\left(B \Sigma_{p^{r}}\right)_{+}} \rightarrow \pi_{0} \boldsymbol{E}^{\left(B \Sigma_{\left.p^{r}\right)_{+}}\right.} / I_{\mathrm{tr}} \stackrel{\alpha_{r}}{\longrightarrow} R
$$

where the power operation $D_{p^{r}}$ arises from the $\mathrm{H}_{\infty}$-ring structure of $\boldsymbol{E}(2.9), I_{\mathrm{tr}}$ is the ideal generated by the images of transfers from proper subgroups of $\Sigma_{p^{r}}$, and $\alpha_{r}$ classifies the subgroup of $i^{*} \mathcal{G}_{E}$ corresponding to $\ell(8.5,2.7)$.

Remark 8.7 In the presence of a level structure as in Definition 8.6, the structure morphism $i$ of $T$ over $S_{E}$ in Definition 8.4 is given by the classifying map

$$
\alpha: A_{0} \stackrel{s_{r}}{\longrightarrow} A_{r} \stackrel{\alpha_{r}}{\longrightarrow} R
$$

from Propositions 4.6 and 5.5, while $\psi_{\ell}^{E}$ is precisely the classifying map

$$
\alpha^{\prime}: A_{0} \stackrel{t_{r}}{\longrightarrow} A_{r} \stackrel{\alpha_{r}}{\longrightarrow} R
$$

(cf. [Rezk2009, Theorem B] for the identification with $t_{r}$ ).

Definition 8.8 (cf. [Ando-Hopkins-Strickland2004, 3.13-3.15]) Let $\boldsymbol{F}:=\boldsymbol{E}^{X_{+}}$and $f: \boldsymbol{E} \rightarrow \boldsymbol{F}$ be the natural map of $\mathrm{H}_{\infty}$-ring spectra. Given any level $A$-structure $\ell: \mathcal{A}_{T} \rightarrow i^{*} \mathcal{G}_{E}$ on $\mathcal{G}_{E}$, let $\ell^{\prime}$ be the unique level $A$-structure on $\mathcal{G}_{F}$ induced by $f,{ }^{13}$ so that the following diagram commutes (with all but the front-left and back-left squares cartesian).

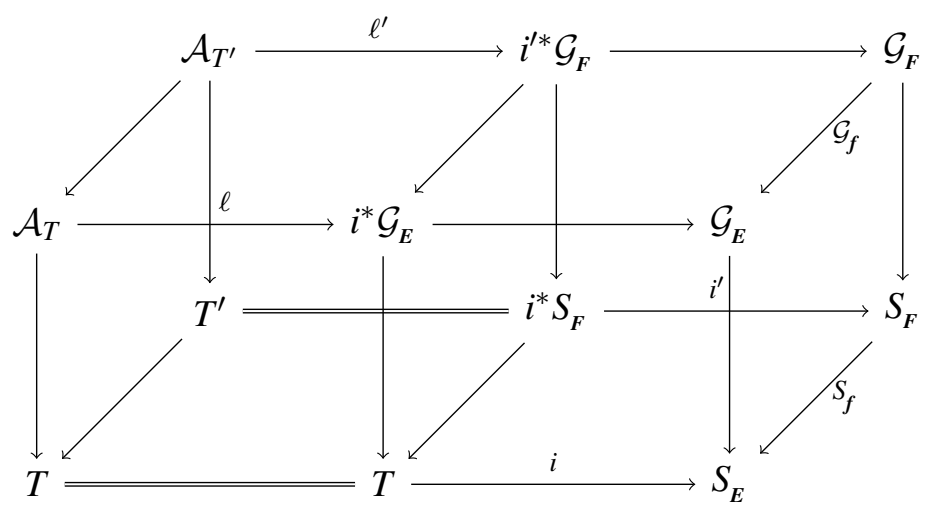

\footnotetext{
${ }^{13}$ Level $A$-structures on $\mathcal{G}_{\boldsymbol{F}}$ are defined analogously to those on $\mathcal{G}_{\boldsymbol{E}}$ as in Definition 8.4.
} 
Let $\psi_{\ell^{\prime}}^{F}: T^{\prime} \rightarrow S_{F}$ be the morphism analogous to $\psi_{\ell}^{E}$ in Definition 8.6, obtained by the naturality of power operations on $\boldsymbol{E}^{0}(X)$. Define $\psi_{\ell}^{F / E}$ to be the unique $T$-morphism which fits into the following commutative diagram.

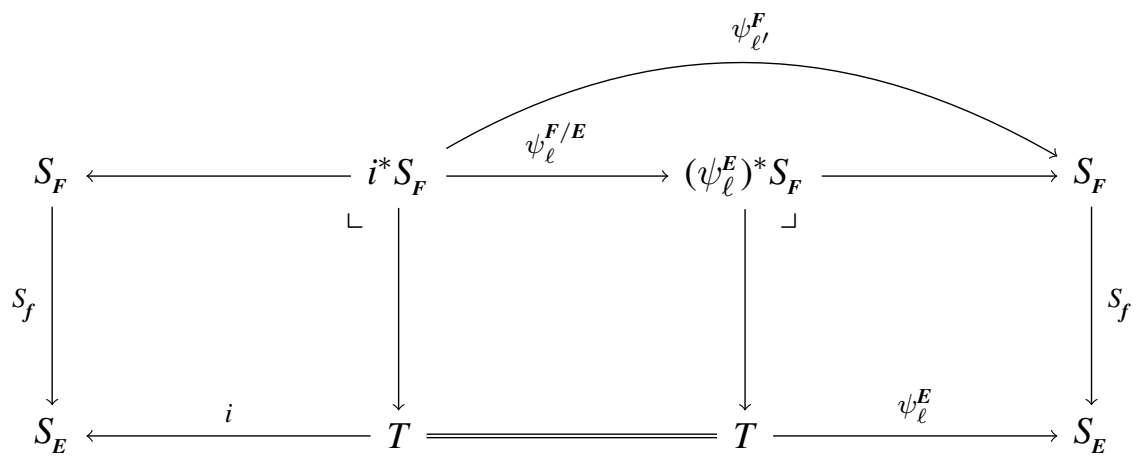

In particular, when $\boldsymbol{F}=\boldsymbol{E}^{\left(\mathbb{C P}^{\infty}\right)_{+}}$, write $\psi_{\ell}^{F / E}$ as

$$
\psi_{\ell}^{\mathcal{G} / E}: i^{*} \mathcal{G}_{E} \rightarrow\left(\psi_{\ell}^{E}\right)^{*} \mathcal{G}_{E}
$$

Remark 8.10 Let $\boldsymbol{F}=\boldsymbol{E}^{\left(\mathbb{C P}^{\infty}\right)_{+}}$. When $A=\mathbb{Z} / p$, the diagram (8.9) lifts (3.6). More generally, let $\mathcal{H} \subset i^{*} \mathcal{G}_{E}$ correspond to $\ell$ as in Remark 8.5. Comparing (8.9) to the universal example (5.7) and Remark 8.7, we see that $\psi_{\ell}^{\mathcal{G} / E}$ is precisely the isogeny $l_{H}$ from (6.9) if we assume without loss of generality that the $\star$-isomorphism (4.7) is the identity.

8.11 Norm maps In view of [Ando-Hopkins-Strickland2004, Theorem 3.25], we have compared above the ingredients that constitute descent data for level structures on $\mathcal{G}_{E}$ - level structures $\ell$, classifying maps $i$ and $\psi_{\ell}^{E}$, isogenies $\psi_{\ell}^{\mathcal{G} / E}$-with corresponding terms from the earlier sections of this paper. There is one more and key ingredient that goes into the condition [ibid., 4.14] for $\mathrm{H}_{\infty} M U\langle 0\rangle$-orientations.

Definition 8.12 (cf. [ibid., Definitions 10.1, 10.9]) Let $\psi: \mathcal{G} \rightarrow \mathcal{G}^{\prime}$ be an isogeny of formal groups with kernel $\mathcal{K}$. Let $\mu, \pi: \mathcal{G} \times \mathcal{K} \rightarrow \mathcal{G}$ be the multiplication, projection maps, and $q: \mathcal{G} \rightarrow \mathcal{G} / \mathcal{K}$ be the quotient map, as in (2.3). Define $N_{\psi}: \mathcal{O}_{G} \rightarrow \mathcal{O}_{G^{\prime}}$ to 
be the horizontal composite

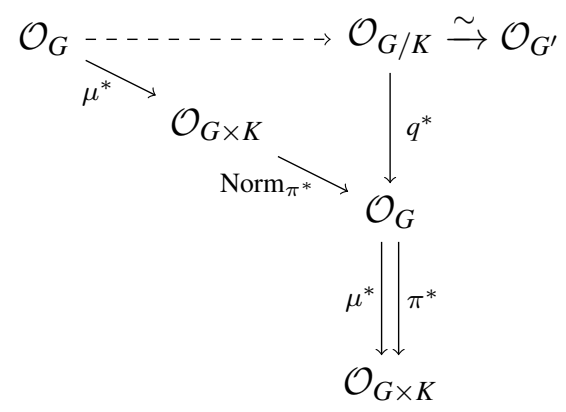

where the vertical maps exhibit $\mathcal{O}_{G / K}$ as an equalizer, Norm $\pi^{*}$ sends $a$ to the determinant of multiplication by $a$ on $\mathcal{O}_{G \times K}$ as a finite free $\mathcal{O}_{G}$-module via $\pi^{*}$, and the factorization through $\mathcal{O}_{G / K}$ was shown, e.g., in [Strickland1997, Theorem 19].

Remark 8.14 Since $q \circ \mu=q \circ \pi$, we have Norm $_{\pi^{*}} \circ \mu^{*}=q^{*} \circ \operatorname{Norm}_{q^{*}}$ (by an argument similar to the proof of the factorization mentioned above). Thus the dashed arrow in (8.13) is Norm $_{q^{*}}$ by uniqueness from the universal property of an equalizer.

Suppose that the isogeny $\psi$ is over a field $k$ of characteristic $p$, and is hence of degree $p^{r}$ for some $r \geq 0$. Comparing [ibid., Theorem 19(i)] and Lemma 3.10, we see that $N_{\psi}$ is precisely the map $\Lambda_{\psi}^{*}$ in (3.9).

Remark 8.15 Let $\psi$ be the isogeny $\psi_{\ell}^{\mathcal{G} / E}: i^{*} \mathcal{G}_{E} \rightarrow\left(\psi_{\ell}^{E}\right)^{*} \mathcal{G}_{E}$ over $R$ from Definition 8.8. Let $x$ be any coordinate on $i^{*} \mathcal{G}_{E}$. In view of Remark 8.5, we have from [Ando-Hopkins-Strickland2004, 10.11] that

$$
\psi^{*} N_{\psi}(x)=\prod_{a \in A} T_{a}^{*}(x)=\prod_{Q \in \mathcal{H}(R)}\left(x \underset{i^{*} G_{E}}{+} x(Q)\right)
$$

where $T_{a}: i^{*} \mathcal{G}_{E} \rightarrow i^{*} \mathcal{G}_{E}$ translates any $R$-point $P$ on $i^{*} \mathcal{G}_{E}$ to $P+Q$, with $Q=\phi_{\ell}(a)$. Comparing this to (6.16), with $\mathcal{F}=i^{*} \mathcal{G}_{E}$, we see that

$$
\psi^{*} N_{\psi}(x)=f_{H}^{*}\left(x_{H}\right)
$$

Now, given any coordinate $s$ on $\mathcal{G}_{E}$, the condition [ibid., 4.14] states that

$$
\left(\psi_{\ell}^{E}\right)^{*} s=N_{\psi_{\ell}^{\mathcal{G} / E}}\left(i^{*} s\right)
$$

Pulling this back along $\psi_{\ell}^{\mathcal{G} / E}$ and writing $x:=i^{*} s$, we get an equivalent identity

$$
l_{H}^{*}\left(x^{\prime}\right)=f_{H}^{*}\left(x_{H}\right)
$$

where $l_{H}=\psi_{\ell}^{\mathcal{G} / E}$ from Remark 8.10, and $x^{\prime}:=\left(\psi_{\ell}^{E}\right)^{*} s=\alpha_{r}^{*} t_{r}^{*} s$ from Remark 8.7. In view of $(6.10,6.11)$, we see that $(8.16)$ is equivalent to $(6.18)$. This shows that [ibid., $4.14]$ and our norm-coherence condition agree (cf. (6.20)). 
Corollary 8.17 Let $\boldsymbol{E}, \mathcal{G}_{E}$, and $\mathcal{G}$ be as in (8.2). Given any coordinate $x_{G}$ on $\mathcal{G}$, there exists a unique coordinate $x$ on $\mathcal{G}_{E}$ lifting $x_{G}$ such that its corresponding $M U\langle 0\rangle$ orientation for $\boldsymbol{E}$ is $\mathrm{H}_{\infty}$.

Proof In view of Remark 8.15, the corollary follows from Proposition 7.1. In particular, as $p$ is not a zero-divisor in $\pi_{0} \boldsymbol{E}$, we may apply [Ando-Hopkins-Strickland2004, Proposition 6.1] for $\mathrm{H}_{\infty} M U\langle 2 k\rangle$-orientations with $k=0$ (cf. [ibid., discussion following 1.6]).

\section{References}

[Ando1992] Matthew Ando, Operations in complex-oriented cohomology theories related to subgroups of formal groups, ProQuest LLC, Ann Arbor, MI, 1992, Thesis (Ph.D.)Massachusetts Institute of Technology. MR2716371

[Ando1995] Matthew Ando, Isogenies of formal group laws and power operations in the cohomology theories $E_{n}$, Duke Math. J. 79 (1995), no. 2, 423-485. MR1344767

[Ando2000] Matthew Ando, Power operations in elliptic cohomology and representations of loop groups, Trans. Amer. Math. Soc. 352 (2000), no. 12, 5619-5666. MR1637129

[Ando-Hopkins-Strickland2001] M. Ando, M. J. Hopkins, and N. P. Strickland, Elliptic spectra, the Witten genus and the theorem of the cube, Invent. Math. 146 (2001), no. 3, 595-687. MR1869850

[Ando-Hopkins-Strickland2004] Matthew Ando, Michael J. Hopkins, and Neil P. Strickland, The sigma orientation is an $H_{\infty}$ map, Amer. J. Math. 126 (2004), no. 2, 247-334. MR2045503

[Ando-Strickland2001] M. Ando and N. P. Strickland, Weil pairings and Morava K-theory, Topology 40 (2001), no. 1, 127-156. MR1791270

[Brun2007] M. Brun, Witt vectors and equivariant ring spectra applied to cobordism, Proc. Lond. Math. Soc. (3) 94 (2007), no. 2, 351-385. MR2308231

[Bruner-May-McClure-Steinberger1986] R. R. Bruner, J. P. May, J. E. McClure, and M. Steinberger, $H_{\infty}$ ring spectra and their applications, Lecture Notes in Mathematics, vol. 1176, Springer-Verlag, Berlin, 1986. MR836132

[Coleman1979] Robert F. Coleman, Division values in local fields, Invent. Math. 53 (1979), no. 2, 91-116. MR560409

[Elmendorf-Kriz-Mandell-May 1997] A. D. Elmendorf, I. Kriz, M. A. Mandell, and J. P. May, Rings, modules, and algebras in stable homotopy theory, Mathematical Surveys and Monographs, vol. 47, American Mathematical Society, Providence, RI, 1997, With an appendix by M. Cole. MR1417719 
[Goerss-Hopkins2004] P. G. Goerss and M. J. Hopkins, Moduli spaces of commutative ring spectra, Structured ring spectra, London Math. Soc. Lecture Note Ser., vol. 315, Cambridge Univ. Press, Cambridge, 2004, pp. 151-200. MR2125040

[Hill-Hopkins2016] Michael A. Hill and Michael J. Hopkins, Equivariant symmetric monoidal structures. arXiv:1610.03114

[Hopkins1995] Michael J. Hopkins, Topological modular forms, the Witten genus, and the theorem of the cube, Proceedings of the International Congress of Mathematicians, Vol. 1, 2 (Zürich, 1994), Birkhäuser, Basel, 1995, pp. 554-565. MR1403956

[Hopkins2002] M. J. Hopkins, Algebraic topology and modular forms, Proceedings of the International Congress of Mathematicians, Vol. I (Beijing, 2002), Higher Ed. Press, Beijing, 2002, pp. 291-317. MR1989190

[Hopkins-Lawson2016] Michael J. Hopkins and Tyler Lawson, Strictly commutative complex orientation theory. arXiv:1603.00047

[Katz-Mazur1985] Nicholas M. Katz and Barry Mazur, Arithmetic moduli of elliptic curves, Annals of Mathematics Studies, vol. 108, Princeton University Press, Princeton, NJ, 1985. MR772569

[Lubin1967] Jonathan Lubin, Finite subgroups and isogenies of one-parameter formal Lie groups, Ann. of Math. (2) 85 (1967), 296-302. MR0209287

[Lubin-Tate1966] Jonathan Lubin and John Tate, Formal moduli for one-parameter formal Lie groups, Bull. Soc. Math. France 94 (1966), 49-59. MR0238854

[May1977] J. Peter May, $E_{\infty}$ ring spaces and $E_{\infty}$ ring spectra, Lecture Notes in Mathematics, Vol. 577, Springer-Verlag, Berlin-New York, 1977, With contributions by Frank Quinn, Nigel Ray, and Jørgen Tornehave. MR0494077

[Mumford2008] David Mumford, Abelian varieties, Tata Institute of Fundamental Research Studies in Mathematics, vol. 5, Published for the Tata Institute of Fundamental Research, Bombay; by Hindustan Book Agency, New Delhi, 2008, With appendices by C. P. Ramanujam and Yuri Manin, Corrected reprint of the second (1974) edition. MR2514037

[Quillen1969] Daniel Quillen, On the formal group laws of unoriented and complex cobordism theory, Bull. Amer. Math. Soc. 75 (1969), 1293-1298. MR0253350

[Rezk2009] Charles Rezk, The congruence criterion for power operations in Morava Etheory, Homology Homotopy Appl. 11 (2009), no. 2, 327-379. MR2591924

[Rezk2014] Charles Rezk, Isogenies, power operations, and homotopy theory, Proceedings of the ICM, Seoul 2014, vol. 2 (2014), 1125-1146.

[Rezk2015] Charles Rezk, Elliptic cohomology and elliptic curves (Part 4), available at https://www . youtube. com/watch?v=r_7SsIoU9No.

[Rezk2016] Charles Rezk, Elliptic cohomology and elliptic curves, available at http://www . math.uiuc.edu/ rezk/felix-klein-lectures-notes.pdf (version: 2016-06-06). 
[Rotman2010] Joseph J. Rotman, Advanced modern algebra, Graduate Studies in Mathematics, vol. 114, American Mathematical Society, Providence, RI, 2010, Second edition [of MR2043445]. MR2674831

[Strickland1997] Neil P. Strickland, Finite subgroups of formal groups, J. Pure Appl. Algebra 121 (1997), no. 2, 161-208. MR1473889

[Strickland1998] N. P. Strickland, Morava E-theory of symmetric groups, Topology 37 (1998), no. 4, 757-779. MR1607736

[Strickland] Neil P. Strickland, Functorial philosophy for formal phenomena, available at http://hopf.math.purdue.edu/Strickland/fpfp.pdf.

[Tambara1993] D. Tambara, On multiplicative transfer, Comm. Algebra 21 (1993), no. 4, 1393-1420. MR1209937

[Walker2008] Barry John Walker, Multiplicative orientations of K-Theory and p-adic analysis, ProQuest LLC, Ann Arbor, MI, 2008, Thesis (Ph.D.)-University of Illinois at UrbanaChampaign. MR2712595

[Zhu2014] Yifei Zhu, The power operation structure on Morava E-theory of height 2 at the prime 3, Algebr. Geom. Topol. 14 (2014), no. 2, 953-977. MR3160608

[Zhu2015a] Yifei Zhu, The Hecke algebra action on Morava E-theory of height 2, available at https://yifeizhu.github.io/ho.pdf.

[Zhu2015b] Yifei Zhu, Modular equations for Lubin-Tate formal groups at chromatic level 2, available at https://yifeizhu.github.io/me.pdf.

Department of Mathematics, Southern University of Science and Technology, Shenzhen, Guangdong 518055 China

zhuyf@sustc.edu.cn 